\title{
System-wide tail comovements: a bootstrap test for cojump identification on the S\&P 500, US bonds and exchange rates.*
}

\author{
Jean-Yves Gnabo ${ }^{\dagger} \quad$ Lyudmyla Hvozdyk ${ }^{\ddagger} \quad$ Jérôme Lahaye $^{\S}$
}

First Version: July 2012. This Version: February 2014.

\begin{abstract}
This paper studies bivariate tail comovements on financial markets that are of crucial importance for the world economy: The S\&P 500, US bonds, and currencies. We propose to study that form of dependence under the lens of cojump identification in a bivariate Brownian semimartingale with idiosyncratic jumps, as well as cojumps. Whereas univariate jump identification has been widely studied in the high-frequency data literature, the multivariate literature on cojump identification is more recent and scarcer. Cojump identification is of interest, as it may identify comovements which are not trivially visible in a univariate setting. That is, price changes can be small relative to local variation, but still abnormal relative to local covariation. This paper investigates how simple parametric bootstrapping of the product of assets' intraday returns can help detect cojumps in a multivariate Brownian semi-martingale with both idiosyncratic jumps and cojumps. In particular, we investigate how to disentangle idiosyncratic jumps from common jumps at an intraday level for pairs of assets. The approach is flexible, trivial to implement, and yields good power properties. It allows to shed new light on extreme dependence at the world economy level.

Keywords: cojump, co-jump, jump, bootstrap, diffusion, Brownian, semi-martingale, high-frequency, simulation, risk, diversification

JEL Codes: C12, C14, C15, C32, C33, C58, F31 ,G12, G14
\end{abstract}

\footnotetext{
${ }^{*}$ The authors wish to thank the Financial Mathematics and Computation Research Cluster $\left(F M C^{2}\right)$ at UC Dublin, David Edelman, as well as $F M C^{2}$ seminar participants for useful comments and discussions.

${ }^{\dagger} \mathrm{CeReFiM}$ and University of Namur

$\ddagger$ University of Essex and CFAP, University of Cambridge

§Fordham University; corresponding author. jlahaye@fordham.edu
} 


\section{Introduction}

The most recent financial crisis has emphasized the risk of extreme comovements. Events such as bailout plan announcements, corporate or government defaults, natural calamities, to name just a few, may create large jumps and cojumps in financial asset prices. But given the continuous flow of economic information, some news may have economy-wide relevance without being as extreme as those just listed, potentially causing smaller cojumps. Having an accurate understanding of the resulting tail comovements in financial markets, and being able to hedge against them is of critical importance.

Whereas univariate jump identification has been widely studied in the high-frequency data literature, the multivariate literature on cojump identification is more recent and scarcer. This paper proposes a cojump test based on a bootstrap procedure in order to investigate whether abnormal comovements exist and can be detected beyond mere simultaneous occurrences of univariate jumps. That is, we investigate whether we can find market comovements which cannot necessarily be identified otherwise with a univariate approach. We study these movements on key markets for the world economy: the S\&P 500, US bonds, and currencies.

To identify abnormal comovements, we study the potential benefits of parametric bootstrapping methods to detect intraday cojumps in two asset prices. Under a multivariate Brownian semimartingale with idiosyncratic jumps and common jumps, we exploit high-frequency data to estimate jump-robust covariance matrices and simulate null distributions of returns' product under different scenarios through a bootstrapping procedure.

The intuition behind our approach is simple. In a univariate setting, when a jumps occurs, the observed intraday return is likely to lie outside the region of returns generated by the diffusive part only. Likewise, in a multivariate setting, when a cojump occurs on two or more asset prices, the observed return product (i.e. in the bivariate case, the intraday return of asset $1 \times$ that of asset 2 ) is likely to lie outside the region of returns product generated by the diffusive part and potentially the idiosyncratic jump part. Therefore, to detect cojumps, we use a simple statistic: the product of observed intraday returns whose distribution under the null is generated through simulations. This approach is an extension of Bollerslev, Law, and Tauchen (2008) to a bivariate setting, and can also be seen as a multivariate extension of Lee and Mykland (2008).

Under the assumption of neither idiosyncratic jumps, nor cojumps, and assuming a Brownian semi-martingale, the distribution of the return product is the normal-product distribution. Under the null of idiosyncratic jumps only, we elaborate on the normal product distribution to include idiosyncratic jumps. The use of these distributions has the advantage of reducing the multivariate jump detection problem to a univariate one that is trivial to implement and simulate.

We pay particular attention to the impact of jumps on cojump detection. Indeed, large idiosyncratic jumps tend to inflate any test statistic up to a level where a cojump could be wrongly detected. We show how simulating idiosyncratic jumps allow to discriminate between common and individual jumps. Moreover, to tackle this issue, we show how the combination of univariate and multivariate techniques can be informative regarding the nature of the discontinuity, i.e. whether it is an idiosyncratic one or a common one.

We illustrate our approach in two empirical applications: we study to what extent stocks (through the S\&P 500 index) and US bonds actually cojump. The empirical question raised in 
this paper is whether abnormal comovement can be detected beyond obvious simultaneous large jumps. This question is important for asset allocation, risk and portfolio management as well as option pricing. A second application investigates abnormal comovements on bonds and currencies. Specifically, the identification of jumps and cojumps in the USD-JPY exchange rate and the US bond returns enables to document the discussion on the so-called currency crashes that has been recently revamped in the literature on carry trade.

The rest of the paper is organized as follows. Section 2 describes the data generating process and a general algorithm to detect cojumps. Section 3 frames this approach within the existing literature. In Section 4, we demonstrate the power and size properties of our approach. Section 5 is the empirical application. Section 6 concludes.

\section{Cojump Detection}

\subsection{Setting}

Our approach can be thought within a standard general bivariate diffusion ${ }^{1}$ (with $n=2$ assets) ${ }^{2}$ with idiosyncratic (also called individual or univariate jumps) and common jumps (i.e. cojumps). That is, we assume an $\mathcal{F}_{t}$-adapted Brownian semi-martingale for the log-price vector $p(t)=\left(p_{1}(t), p_{2}(t)\right)^{\prime}$ defined on the 2 -dimensional probability space $\left(\Omega, \mathcal{F}, \mathcal{F}_{t}, \mathbb{P}\right)$ :

$$
\begin{aligned}
& d p_{1}(t)=\mu_{1}(t) d t+\sigma_{1}(t) d W_{1}(t)+\kappa_{1}(t) d q_{1}(t)+\kappa(t) d q(t) \\
& d p_{2}(t)=\mu_{2}(t) d t+\sigma_{2}(t) d W_{2}(t)+\kappa_{2}(t) d q_{2}(t)+\kappa(t) d q(t),
\end{aligned}
$$

where, for $i=1,2, p_{i}(t)$ is the $\log$ price, $\mu_{i}(t)$ is the drift, $\sigma_{i}(t)$ is the local volatility process, $d W_{i}(t)$ is a Brownian motion increment. Brownian increments are linked through a correlation coefficient $\rho(t)$ and all variables are well-behaved with usual assumptions. Notably, variance-covariances are $\mathcal{F}_{t}$-adapted càdlàg and bounded away from zero almost surely. Three jump components are present: two idiosyncratic jump processes $\kappa_{1}(t) d q_{1}(t)$ and $\kappa_{2}(t) d q_{2}(t)$, as well as a cojump process $\kappa(t) d q(t)$. $d q_{i}(t)$ and $d q(t)$ are counting process increments, while $\kappa_{i}(t)$ and $\kappa(t)$ are jump sizes. Our goal is to identify $\kappa(t) d q(t)$ through simulations, and thereby describing the conditions under which it can be identified.

For that purpose, we use discretely observed intraday returns ${ }^{3}$ :

$$
r_{i, t, j}=p_{i}\left(t-1+\frac{j}{M}\right)-p_{i}\left(t-1+\frac{j-1}{M}\right)
$$

\footnotetext{
${ }^{1}$ Nothing prevents to discretize the model and consider alternative distributions such as multivariate students. We leave this question for future research.

${ }^{2}$ Eventhough nothing prevents to consider higher dimensional issues, this paper focuses on the bivariate case for several reasons: first, the $\mathrm{N}$-asset case is studied elsewhere (see e.g. Bollerslev, Law, and Tauchen (2008) or more recently Boffelli, Novotny, and Urga (2014)). Second, the bivariate case is particularly challenging, as the univariate jump influence on cojump statistic tend to create size issue. Jacod and Todorov (2009) propose a bivariate test. Our study complements theirs in that purpose. Third, the bivariate case is of economic and financial interest in diverse applications, notably those developed in this paper.

${ }^{3}$ In this paper, we ignore microstructure noise by sampling sparsely at usual frequencies of about 15-minutes. Microstructure noise can be simulated in a similar setting, however. To save space and focus on the issue of disentangling jumps from cojumps, we leave the issue of the link between noise and cojump detection for future research.
} 
for firm $i$, intraday period $j$ and day $t$. There are $M$ intraday returns over one day and $M+1$ observed prices: $p_{i}(t-1), p_{i}\left(t-1+\frac{1}{M}\right), \ldots, p_{i}(t)$.

Using these returns, we can estimate daily variance using the widely used realized variance estimator:

$$
R V_{i, t}=\sum_{j=1}^{M} r_{i, t, j}^{2}
$$

or one jump-robust counterpart, bipower variation:

$$
B V_{i, t}=\mu_{1}^{-2}\left(\frac{M}{M-1}\right) \sum_{j=2}^{M}\left|r_{i, t, j-1}\right|\left|r_{i, t, j}\right|,
$$

with $\mu_{1}=\sqrt{2 / \pi}$. We have that under a Brownian semimartingale with jumps (Barndorff-Nielsen and Shephard 2004, 2006):

$$
\lim _{M \rightarrow \infty} B V_{i, t}=\int_{t-1}^{t} \sigma_{i}^{2}(s) d s .
$$

Assuming constant variance throughout a given day, Lee and Mykland (2008) test for jumps estimating integrated volatility over an intraday period, $\int_{t-1+\frac{j-1}{M}}^{t-1+\frac{j}{M}} \sigma_{i}^{2}(s) d s$, using $B V_{i, t} / M$. They test for idiosyncratic jumps using

$$
\frac{\left|r_{i, t, j}\right|}{\sqrt{B V_{i, t} / M}}
$$

which should be a realization from the absolute normal distribution under no jumps. ${ }^{4}$ We use this approach subsequently when testing for jumps in a univariate setting.

Lahaye, Laurent, and Neely (2011) use Lee and Mykland's (2008) test to identify cojumps, defined as jumps occurring simultaneously on different markets. In the remainder of the text, we refer to this approach using the oxymoron "univariate cojumps" or "LM-cojumps", as this detection relies on a univariate jump detection with the Lee and Mykland (2008) approach. In the next subsection, we introduce the statistic used to identify cojumps, explicitly accounting for the asset's covariation. We refer to this approach as "bootstrap cojumps". Through a simulation exercise in Section 4, we show under what circumstances "univariate cojumps" or "bootstrap cojumps" are more appropriate, and whether the "bootstrap cojumps" approach allows to detect cojumps that would remain invisible otherwise.

\subsection{Cojump Test}

To detect a cojump at time $t, j$, we consider the following statistic: the product of intraday returns across $n=2$ assets at that time. That is,

$$
\operatorname{coj}_{t, j}=\prod_{i=1}^{n} r_{i, t, j} .
$$

Under the null of no jump and no cojump, this statistic follows a normal product distribution. This distribution has a non-standard form. The first results on this distribution date back to the 1930's. Craig (1936) is the first to find the moment generating function of the product of two normal variables in algebraic form. Aroian (1944) showed theoretically under what condition the

\footnotetext{
${ }^{4}$ More precisely, Lee and Mykland (2008) consider the distribution of the sample maximum of that statistic.
} 
normal product distribution can be approximated by a Normal distribution. More recently, Ware and Lad (2003) discuss three methods to approximate the normal product: numerical integration, Monte Carlo simulation, and normal approximation analytical results. They find that the first two methods, numerical integration and Monte Carlo approximation, yield similar results. The latest method, the normal approximation, lacks the generality required in our application. Indeed, the quality of the approximation depends on the relative size of the means compared to the variances. The larger the mean to variance ratio, the better the normal approximation to the normal product.

This leads us to chose the Monte Carlo method to generate the product distribution under no jumps and cojumps. This choice is further motivated by the need to have a flexible and consistent framework that allows to go beyond the normal product to include idiosyncratic jumps. We expect that when a cojump occurs, the return product containing the cojump should not lie in the region of the product of a return generated by the diffusive part multiplied by a return containing an idiosyncratic jump.

The intuition behind the product distribution is simple: a high frequency return product should be small in the absence of jumps-cojumps. It is also expected to be small in the presence of an idiosyncratic jump, as the other jump-free return will downscale the statistic. However, in the presence of a cojump, the statistic should explode. Of course, it is possible that a very large idiosyncratic jump inflate the product to a level where a cojump would be detected. We will study the possibility of such misclassification by combining the multivariate inference with the information obtained from a univariate analysis.

To sum up, given local covariation and jump conditions, it is easy to simulate the return product under the null of no jumps and cojumps, and under the null of no cojump. We explain hereunder how we estimate the local moments to generate these distributions.

\subsection{General Algorithm}

To generate null distributions, we estimate robust-to-jumps local moments. Our general algorithm proceeds as follows. For each intraday period $(t, j)$ :

1. Estimate intraday (e.g. 5-minute or 15-minnute) integrated volatilities for all assets $(i=$ $1, \ldots, n): \sqrt{\int_{t, j-1}^{t, j} \sigma_{i}(s) d s}$, using a univariate robust-to-jump measure.

2. For a pair of assets $i$ and $h$, estimate $\rho_{t}^{i, h}$, the integrated correlation.

3. Estimate or hypothesize an idiosyncratic jump size.

4. With the parameters estimated in $1-3$, simulate the distribution of $\operatorname{coj}_{t, j}$ under idiosyncratic jumps.

5. Choose a significance level, and check whether the observed $\operatorname{coj}_{t, j}$ falls beyond the corresponding critical value. Alternatively, build a variable expressing the degree of cojump belief.

Concerning step 1, the approach taken by Lee and Mykland (2008) is to use daily bipower variation (see Section 2.1). This assumes constant volatility throughout the day, such that that the daily measure can be scaled to the intraday interval. In this paper, we adopt the same approach. Step 2 requires the choice of a covariance estimator. As developed in the subsequent sections, we will test three different estimators. 
For step 3, real data provides estimates for jump parameters, applying for example Lee and Mykland's (2008) test. On the other hand, running tests with a deterministic hypothesized jump size might provide a good indication as to whether we have a cojump or not. That would allow to infer if a cojump is present given an idiosyncratic jump in one asset. Therefore, this provides a sense of the plausibility of a cojump vs. a jump. In that sense, simulating the jump occurrence randomness in the bootstrap is not a necessity. A conservative way to approach the problem consists of generating the distribution of the returns' product under idiosyncratic jumps, given a jump occurrence. So when generating that distribution, we will include a jump in every draw.

Once local jump and covariation parameters are estimated (or hypothesized), it is trivial to use random number generators to draw returns from a $n$-variate Brownian motion with jumps and generate the distribution of the product of returns.

In the next section, we discuss how this approach relates to the literature.

\section{Literature}

The univariate non-parametric jump literature is broad. ${ }^{5}$ Univariate tests are evaluated through Monte Carlo procedures in Dumitru and Urga (2012). Dumitru and Urga (2012) study point to the excellent properties of the intraday approach of Lee and Mykland's (2008) test. On the other hand, the multivariate literature is much scarcer. As for univariate jumps, the multivariate literature can be divided between intraday cojump identification and daily (or aggregate) cojump identification which allows to tell whether one day contains a cojumps without identifying its precise occurrence time. For the former approach,Bollerslev, Law, and Tauchen (2008) propose to use the mean cross product of returns of a large number of assets, as that quantity should be sensitive to cojumps while diversifying away the idiosyncratic jump influence. For the latter approach, Jacod and Todorov (2009) use the properties of ratios of power variations estimated at different sampling frequencies. After a univariate jump detection on two series, they test whether these jumps are joint or disjoint, that is idiosyncratic or common.

Our approach can be seen as an extension Bollerslev, Law, and Tauchen (2008) to $n$ assets (including small $n$, and in our particular case, the smallest possible, i.e. $n=2$ ) through straightforward elaboration of a parametric bootstrap procedure. It is also in a sense, a multivariate extension of Lee and Mykland (2008), as we use robust local moment estimation to detect cojumps. Finally, our approach aims to detect potential cojumps which are not obvious through a univariate jump detection, which is not the goal of Jacod and Todorov (2009) as they test the null of joint or disjoint jumps given jumps have been detected with a univariate test. Our approach deals with the question whether non (univariate) jump observations might in fact be cojumps.

Our approach relates closely to Bollerslev, Law, and Tauchen (2008), but with substantial difference however. Indeed, Bollerslev, Law, and Tauchen's (2008) portfolio approach is designed to detect cojumps in a large number of assets, whereas our approach is intended to work with 2

\footnotetext{
${ }^{5}$ It encompasses, non exhaustively, Barndorff-Nielsen and Shephard (2006), Lee and Mykland (2008), Andersen, Bollerslev, and Dobrev (2007), Andersen, Dobrev, and Schaumburg (2008), Jiang and Oomen (2008), Aït-Sahalia and Jacod (2006), Aït-Sahalia (2004), Mancini (2009), Podolskij and Ziggel (2010), Corsi, Pirino, and Reno (2010).
} 
assets or more. They consider the following mean cross product ( $m c p)$ statistic:

$$
m c p_{t, j}=\frac{2}{n(n-1)} \sum_{i=1}^{n-1} \sum_{l=i+1}^{n} r_{i, t, j} r_{l, t, j}
$$

and bootstrap a studentized version:

$$
z_{m c p, t, j}=\frac{m c p_{t, j}-\overline{m c p_{t}}}{s_{m c p, t, j}} .
$$

It is clear that the statistic $m c p_{t, j}$ is reduced to $c o j_{t, j}=r_{1, t, j} \times r_{2, t, j}$ in the case of $n=2$ assets. But whereas Bollerslev, Law, and Tauchen (2008) use a large $n$ to diversify away the jump influence, we account for jump impact by incorporating jumps in the bootstrapping procedure. Furthermore, we incorporate univariate test for further information regarding the nature of the discontinuity.

Our approach can also be seen as a multivariate extension of Lee and Mykland (2008) in the sense that we consider a cojump as a large product realization with respect to local covariation. Lee and Mykland (2008) detect jump when a return is large with respect to local variation. Another way of considering a multivariate extension of Lee and Mykland (2008) is through multivariate studentization of returns. This approach is taken by Ju (2010) and can be seen as an outlier detection using the Mahalanobis distance. The latter approach, however, tests the null of no jumps against a jump in at least one asset. So it is not suited for our purpose. Ju (2010) considers the feasible statistic

$$
L_{t, j}=r_{t, j}^{\prime} \widehat{\Sigma_{t, j}^{-1}} r_{t, j}
$$

where $r_{t, j}$ is the $n \times 1$ asset return vector and $\widehat{\Sigma_{t, j}}$ is the robustly estimated covariance matrix. Under the null of no jump-cojump, $L_{t, j}$ follows a $\chi^{2}$ distribution. Nevertheless, $L_{t, j}$ is a sum of functions of squared returns and paired products. It is therefore sensitive to jumps and cojumps. Our approach, on the other hand, focuses on returns product, which should be large when a cojump occurs and small otherwise.

Jacod and Todorov (2009) test for common jumps in two asset prices with a daily approach. This test is more directly comparable to ours as it works with two assets. Jacod and Todorov (2009) use the ratio of power variation estimators evaluated at two different frequencies. They also provide infill asymptotic results for a statistic to test the null of no cojumps (that is, the null of idiosyncratic jumps), given jumps on a given day. Because our approach is also bivariate, our approach complements their. Nevertheless, there are also substantial differences, as the null hypothesis is defined differently, and we work intradaily.

Two recent studies also deal with cojump identification. Gobbi and Mancini (2012) apply thresholding techniques to derive a cojump robust covariance estimator. They provide a central limit theorem and a cojump test. But their analysis is essentially aggregated (as Jacod and Todorov (2009)), in the sense that they estimate sums of cojumps over a day through the difference between realized covariation and their robust counterpart. As our approach is intradaily, it does not compare directly either to this approach.

Boffelli, Novotny, and Urga (2014) propose to study cojumps by measuring the degree of commonality of jumps across asset during a period of time such as a month. They detect jumps with univariate techniques Lee and Mykland (2008) and measure common feature in the style of Engle and Kozicki (1993). 
Finally, we note that even though we do not deal with a state-space model, the type of algorithm we propose presents similarities with the sequential Monte Carlo approach proposed initially by Gordon, Salmond, and Smith (1993) and applied in Jondeau, Lahaye, and Rockinger (2013), i.e. the seminal bootstrap particle filter. That is, in the sense that our algorithm is online, and amounts to generate conditional distributions in which we gauge the plausibility of a realization, in a way that can be seen as parallel to propagating particles and attaching weights to them.

The next section runs a Monte Carlo analysis to illustrate the extent to which we can identify cojumps.

\section{Monte Carlo Evaluation}

\subsection{Null and Alternative Distributions}

We perform a Monte-Carlo simulation exercise to evaluate our approach. In what follows, we define the considered DGP. For each Monte-Carlo experience, we simulate the system ( $n=2$ assets) given in Eq. 2.1 with the parameters given in Table 1. That is, we generate $K=21600$ points per day, for 100 days.

At each simulated point, two returns are drawn from a bivariate normal distribution with a correlation coefficient $\rho$ in $\{-0.7,-0.5,-0.3,-0.1,0.1,0.3,0.5,0.7\}$, and volatilities $\sigma_{1}$ and $\sigma_{2}$ at 0.0001 or 0.0002 . That yields annualized volatilities of $0.0001 \times \sqrt{200 \times 21600} \simeq 0.2$ or $0.0002 \times$ $\sqrt{200 \times 21600} \simeq 0.4$, i.e. about $20 \%$ or $40 \%$. Figure 1 shows one realization of 21600 points, i.e. one day of trading. For our applications, we will sample these prices at a frequency of $\Delta=5$ minutes, yielding $M=6 * 60 / \Delta=72$ intraday intervals and $O=K / M$ simulated prices per intraday interval.

Cojumps are visible on Figure 1. To produce that path, we have set a cojump intensity $\lambda=3$, meaning that 3 cojumps per day would occur on average in such a setting. For that path, the cojump size is a normal draw with mean 0.01 and standard deviation equal to 5 times the average of $\sigma_{1}$ and $\sigma_{2}$. This is a modest size jump, given the other parameters, as can be seen on Figure 2 that shows the same series but sampled every five minutes. On that figure, cojumps are much less visible than in Figure 1 that shows every simulated point (1 every second during 6 hours). Therefore, one might expect such jumps to be difficult to detect.

We consider 3 scenarios (described in Table 1) for true jumps/cojumps occurrences in the DGP. In the first case, we simulate only cojumps. In scenario 2 , we simulate only idiosyncratic jumps. In the third scenario, no jumps/cojumps are simulated. Jumps and cojumps intensity is set at $\lambda=3$, that is 3 jumps/cojumps per day, on average. In every scenario, jump sizes are deterministic, but chosen in $\{0.004,0.005,0.01\}$. These are tick jump sizes. Compared to 5 -minute integrated volatility $0.0001 * \sqrt{O} \approx 0.0017$ (considering $O=300$ observation per intraday interval $\left.\sigma_{1}=0.0001\right)$, these sizes are small. For example, a jump of 0.004 is hardly twice the 5-minute integrated volatility.

\subsection{Size and Power}

We now adopt the point of view of the econometrician observing prices at discretely sampled intervals. We propose a cojump detection algorithm and demonstrate its power and size properties. 
We also describe under what conditions cojump detection becomes more difficult, i.e. the impact of relative idiosyncratic jump and cojump sizes.

Our algorithm (a particular case of the general algorithm of Section 2.3) proceeds as follows. For each sample day $t$, we assume a DGP with constant variances and correlation throughout the day. However, we allow jump size to vary every intraday period. We generate the null distribution under a conservative idiosyncratic jump size equal to the largest standardized intraday return of the tested pair.

We then follow Brandt and Diebold (2006) no-arbitrage argument to obtain covariance estimates as a function of univariate variation measures and use bipower variation as local volatility estimators.

More precisely, our algorithm proceeds as follows, for each intraday period $(t, j)$ :

1. Estimate 5-minute integrated volatilities $\sqrt{\int_{t, j-1}^{t, j} \sigma_{1}(s) d s}$ and $\sqrt{\int_{t, j-1}^{t, j} \sigma_{2}(s) d s}$ using $\hat{\sigma}_{i, t, j}=$ $\sqrt{\frac{B V_{i, t}}{M}}$, with $B V_{i, t}$ defined in Equation 2.4.

2. Estimate $\rho_{t, j}^{1,2}$, the five minute integrated correlation as a function of $B V_{i, t}$, defined above, and $B V_{t}$, the estimated integrated volatility of a portfolio composed of assets 1 and 2 with weights $w_{1}$ and $w_{2}$ (Brandt and Diebold 2006): $c \hat{o} v_{t, j}^{1,2}=\frac{1}{2 w_{1} w_{2}}\left(B V_{t}-w_{1}^{2} B V_{1, t}-w_{2}^{2} B V_{2, t}\right)$ and $\hat{\rho}_{t, j}^{1,2}=\frac{c \hat{o} v_{t, j}^{1,2}}{\hat{\sigma}_{1, t, j} \hat{\sigma}_{2, t, j}}$.

3. Include an idiosyncratic jump in the asset $i$ that that has the largest absolute standardized return (i.e. according to $\left.\max \left(\frac{\left|r_{1, t, j}\right|}{\sqrt{B V_{1, t} / M}}, \frac{\left|r_{2, t, j}\right|}{\sqrt{B V_{2, t} / M}}\right)\right)$. Then set the jump size equal to the standardized return $\frac{r_{i, t, j}}{\sqrt{B V_{i, t} / M}}$.

4. With the parameters estimated in 1-3, bootstrap the distribution of $\operatorname{coj}_{t}$ under idiosyncratic jumps.

5. Choose a nominal size, and test whether the observed statistic $\operatorname{coj}_{t}$ falls beyond the corresponding critical value.

We use the simulated DGP settings described in Table 1, sample every five minutes and apply our algorithm to detect cojumps. Note that the simulated integrated volatility over 5 minutes are $\sqrt{300} \times 0.0001 \simeq 0.0018$ and $\sqrt{300} \times 0.0002 \simeq 0.0035$. Therefore, $\mathrm{a}($ co)jump of size 0.01 is a relatively small size jump, as is illustrated in Figure 2.

Table 2 reports power and size properties of the bootstrap test. This benchmark simulation uses a nominal size $\alpha_{c o j}=0.0001$. For the different correlations and different jump sizes, we report (in the "multivariate" section of the table) the power (percentage of correct cojump detection) of the bootstrap test under scenario 1 (only cojumps are present), as well as the size (percentage of wrong rejection of the null) under the three scenarios (only cojumps, only jumps, no jumps/cojumps at all).

The "univariate" section of Table 2 provides a mean of assessing the contribution of accounting for correlation in the multivariate approach. We report the power and size of Lee and Mykland's (2008) test when applied to asset 1 and 2 separately under scenario 1 . That is, the columns labeled "power 1" and "power 2" report to what extent the Lee and Mykland (2008) test detects the generated cojumps as a jumps in each asset (together with the respective sizes in columns "size 1" 
and "size 2"). We also report the power and size of using Lee-Mykland simultaneous jump detection as a cojump dection, what we call the "univariate cojumps" (column "power coj"). Finally, for completeness, we provide the power and size of the Lee-Mykland test when applied to the asset containing jumps in scenario 2 (under which a jumps occur only in asset 1).

To compare the size of of the bootstrap test using $\operatorname{coj}_{t}$ (left panel) with the univariate cojump approach (right panel), the latter jump test uses a nominal size of $\alpha_{j}=0.01$ such that the probability of a type-I error is 0.0001 when applied twice independently on both assets.

Table 2 reports results for DGP volatilities $\sigma_{1}$ and $\sigma_{2}$ set at 0.0001 . Table 3 doubles $\sigma_{1}$ or $\sigma_{2}$ to a level of 0.0002 . Increasing volatility is equivalent to reducing the jump size in the asset where volatility is increased.

\subsubsection{Power}

Our findings confirm the intuition according to which small-sized cojumps can be detected when they stand out local covariation. That is, with bootstrap test, we detect very small positive jumps when assets are negatively correlated. In general, power increases and tends to 1 when, for positive jumps, correlation tends to -1 (see column labeled "power" in the left panel "Multivariate bootstrap cojump"). Moreover, for any given correlation, power increases with jump sizes. For example, for 2 negatively correlated assets (with $\rho=-0.5$ ), $42.55 \%$ of the smallest jumps (with size $=0.004$ ) are correctly detected. A slightly larger cojump size $(=0.005)$ for that correlation level (-0.5) leads to a power of about 60 percent. When correlation becomes positive and tends to 1 , small positive cojumps tend to be difficult to detect as they are "drawn" in the normal covariation. This is analog to detecting small jumps when volatility is high. However, when jump size is large, power remains good, even for large positive correlation. For example, about $80 \%$ of cojumps are detected when their size is set at 0.01 , with a correlation $\rho=0.7$.

\subsubsection{Size}

We find a reasonable size in every scenario, though above the nominal size in some instances (see columns labeled "size" in the left panel "Multivariate bootstrap cojump"). However, the presence of univariate jumps (see scenario 2 in the left panel) naturally tends to increase the size. The larger the jump size, the greater the size increase. Nevertheless, the size of the bootstrap test in scenario 1 (column labeled "scenario1/size" in the left panel) is everywhere smaller than the size of the univariate cojump test (see column labeled "scenario1/size coj." in the right panel "Univariate jump/cojump").

\subsubsection{Univariate Tests}

We now turn more specifically to the right panel of 2. Our simulation allows to test what can be detected under cojumps (scenario 1) when assets are considered in isolation. The univariate test we apply (Lee and Mykland 2008) is among the best for such setting (Dumitru and Urga 2012). Therefore, it provides useful benchmark to gauge the interest of going multivariate. As an example, let us consider a jump size of 0.5 and a correlation of -0.7 . In this case, the univariate jump test detects about $65 \%$ of jumps on asset 1 , and $62 \%$ on asset $2 .{ }^{6}$ This means that about $35-38 \%$ of

\footnotetext{
${ }^{6}$ Recall that the chosen size here is $1 \%$ such that cojump tests are directly comparable.
} 
the simulated cojumps remain totally invisible to a jump test (not even detected as a jump in one asset). However, the bootstrap cojump test detects $86 \%$ of the simulated cojumps leaving only $14 \%$ undetected. Moreover, looking at simultaneous jump occurrence (univariate cojumps test) yields a power of about 33\%, much below what we achieve with the multivariate bootstrap test. The sizes for the univariate tests in assets 1 and 2 evolve around their nominal size of $1 \%$. While the size for the univariate cojump test is above the size of the multivariate bootstrap test under scenario 1 .

\subsubsection{Varying Volatility}

Table 3 shows power and size when volatility increases. This is equivalent to producing jumps of relatively smaller sizes. Therefore, We observe a deterioration of power when volatility increases while jump sizes remain constant.

We conclude from this analysis that univariate tests are satisfactory and best to detect jumps and cojumps as long as the jumps size are sufficiently large and have the same sign as the assets' correlation. Nevertheless, to detect small jump comovements in the opposite direction of the correlation, the proposed cojump detection is best.

\section{Applications}

In this section, we propose two empirical applications of the bootstrap cojump test. Each application is based on long time series, covering the recent financial crisis. Table 4 describes the series.

First, we analyze the occurrence of cojumps between bonds and stocks returns, which is of particular importance for asset allocation and portfolio management. Second, intraday cojumps are identified to document the discussion on "currency crashes" (Brunnermeier, Nagel, and Pedersen 2008): we study bonds yields and exchange rates cojumps. For both pairs, we identify cojumps with the bootstrap test, but also jumps with the Lee and Mykland (2008) test. As for the simulation, we compare the bootstrap test and the univariate cojump test. Tables 5 to 7 describe jump/cojump statistics.

Table 5 presents statistics on detected cojumps applying the general algorithm of Subsection 2.3 , set similarly to the algorithm of the simulation exercise (Section 4). That is, we estimate realized covariance using the no-arbitrage approach argument of Brandt and Diebold (2006) and using robust to jump realized measures, i.e. bipower variation. As described above, we assume stable correlation throughout the day, but we allow intraday jump variation (which implies that we generate a null distribution at every intraday observation).

In order to generate the bootstrap distribution of the cojumps statistics, we consider two null hypotheses. The first conservative approach (reported in the lines labeled "with j." of Table 5) considers that a jump occurs in each intraday interval with a size of the magnitude of the largest absolute standardized return. This approach amounts to raise the following question: given a jump occurs in one asset, is the other asset's return large enough such that we can infer a cojump? As an alternative, we consider the case where no jump is simulated (reported in the lines labeled "w/o jumps" of Table 5), which will produce the narrowest null distribution (as including jumps has the 
effect of making extreme realizations of the return product more likely).

Equipped with local moments, we can generate the return product and extract the quantiles from that bootstrapped distribution. For this application, we choose a size of $\alpha_{c o j}=0.0001$ per intraday period (as in the simulation). That is, we expect to detect one spurious cojump out of every 10000 observations. The univariate test size is fixed such that the type-I error probability for cojumps detected with the simultaneous jumps is similar (i.e. $\alpha_{j}=0.01$ ). As often done in the literature, we chose 15-minute frequency for cojumps detection in order to minimize the risk of microstructure noise.

Table 6 and Table 7 differ from Table 5 in the covariance estimation approach. Table 6 still relies on the no-arbitrage approach of Brandt and Diebold (2006) but uses the non-robust realized volatility (RV) as opposed to bipower variation (BV). On the other hand, Table 7 reports results for a robust covariance estimation (the ROWCov of Boudt, Croux, and Laurent (2011a)) that does not rely on a no-arbitrage approach. Results for these alternative covariance estimators (Tables 6 and 7) are discussed in Section 5.3.

We account for the presence of intraday periodicity in asset prices returns. As shown in Boudt, Croux, and Laurent (2011b) for the univariate case, ignoring this feature can lead to severe bias in jump detection. Consequently, we extend the approach proposed by Boudt, Croux, and Laurent (2011b) to the multivariate case in order to control for intraday coperiodicity. That is, each jump (resp. cojump) test in the next subsections is based on periodicity (resp. coperiodicity) corrected returns (resp. return product). The periodic component is a robust scale measure (the weighted standard deviation) obtained for each intraday period using the variation across days. For example, the periodic component for intraday period 1 is a scale estimated with the first return of each day. To compute that scale, returns are preliminarily standardized by their corresponding daily volatility using bipower variation. We refer to Boudt, Croux, and Laurent (2011b) for full details about this estimation. The same procedure is applied to return's product to identify potential coperiodic effects. We find that, as known in the literature, strong periodic effects are present. However, coperiodicity is much milder, almost innocuous. This can be observed on Figures 7 (resp. 8), that report boxplots of returns (resp. returns'product) for each intra-day period (the boxplots are obtained on returns previously standardized by their corresponding scaled daily bipower variation). Where as the scale clearly varies across intraday period in the univariate case (Figure 7), it is rather flat when considering the returns' product (Figure 8). We note however the importance of using a robust approach to identify the (co)periodic component, as non-robust estimation (not reported here) would be heavily influenced by the presence of outliers (that can be observed around the end of the day, in the coperiodicity case, on Figure 8).

\subsection{Stocks and Bonds}

As described in Table 4, our sample ranges from January 42005 to January 10 2013, yielding 2027 working days. Original intraday tick data are obtained from Tick Data for S\&P 500 (SP500) futures and 30-year Treasury Bonds (Bond). The contracts are traded at the Chicago Mercantile Exchange (CME) and the Chicago Board of Trade (CBOT), respectively. Tick data are sampled at the 15-minute frequency using the Tickdata toolbox. The CME and CBOT opens at 09:30-16:15 (Eastern Standard Time) and at 08:20-15:00, respectively. The two markets have overlapping trad- 
ing sessions from 09:30-15:00. Therefore, we compute the 15-minute returns on the S\&P 500 and the 30-year Treasury Bond during 09:30-15:00 each day. We then estimate the stock and bond returns realized covariances, realized variances, jumps and cojumps. As usually done in the literature, we omit the trading days with too many missing values or low trading activity because they will provide poor estimates of volatility. Finally, the sample contains 1963 observation days.

\subsubsection{Correlation}

A large literature assesses the nature of stocks and bond comovements: are these prices comoving in the same or in opposite direction and with what strength. Traditional approaches rely on rolling windows estimations over historical daily data or alternatively, on more sophisticated multivariate GARCH models, such as DCC-GARCH. In general, evidence shows that the two asset classes are correlated (see Engle (2002), Baur and Lucey (2009), Yang, Zhou, and Wang (2009), or Baele and Inghelbrecht (2010)). However, differences in sign and magnitude are usually observed depending on the macroeconomic and financial environments (Baele and Inghelbrecht (2010), Guidolin and Timmermann (2006)). To better accommodate the time varying nature of stock and bond returns correlations, more recent contributions have proposed to use non parametric techniques that exploit the information content of intraday data. Using realized correlation measures at the 5-minute frequency, Aslanidis and Christiansen (2010) show that comovements can be highly volatile, especially in periods of uncertainty in financial markets. However, in presence of cojumps, the realized correlation is a noisy measure of the true correlation.

Figures 3-5 display intradaily returns, daily realized volatilities and daily realized correlations from 2005 to 2013 . The daily realized correlations estimates range from -1 to 1 , with significant differences depending on the sample period. Most of the estimates are concentrated around zero in the first part of the sample. In the aftermath of the financial crisis, the average correlation falls to -0.6 before stabilizing around -0.3 from 2008 onwards. This confirms results from the literature based on former crisis episodes on the link between economic turmoil and stocks and bonds comovements. In particular, Engle (2002) shows that the Dow Jones index and the US bond returns correlations experienced a drastic fall after the Asian crisis, reaching a record low at -0.55 in 1998 from 0.75 in 1995. Those negative correlations observed between stock and bond returns are generally ascribed to a "flight-to-quality" or "flight-to-safety" phenomenon.

\subsubsection{Cojumps}

We now turn to jump and cojump detection statistics in Table 5 (upper-panel for the SP-Bonds pair). Table 5 (using Brandt and Diebold (2006) with bipower variation) reports (for each bootstrap setting, with and without jumps) the number of observations (Obs), the number of detected bootstrap cojumps (coj.), the number of same sign cojumps (same sign) and opposite sign cojumps (opposite sign), the number of univariate jumps in the first series (uni. j. 1) and in the second series (uni.j.2), the number of univariate cojumps, i.e. univariate jumps occurring at the same time (simult j.), the number of cojumps detected by both approaches, i.e. the intersection of the columns 'coj' and 'simult. j.' (common coj.), and finally the number of detected cojumps where 
only one univariate jump was detected (coj. and j.).

Table 5 shows that the univariate jump test rejects the null of no jumps in several occasions. 1039 and 1034 jumps are detected for the S\&P 500 and bonds respectively. With a nominal size of 0.01 per observation, we expect a wrong detection for every 100 observations that is 400 wrongly detected jumps over the sample. Our results therefore strongly support the presence of jumps in both series. Among the 2073 jumps, only a small fraction, i.e. 137, occur simultaneously.

Column 2 of Table 5 displays the number cojump detected, based on the bootstrap test. We find relatively many cojumps. Table 5 reports 161 detected cojumps for the SP500-Bond pair, with the deterministic jump approach. Ignoring jumps (normal-product case) yields 262 cojumps. Considering the chosen size of 0.0001 , we would expect about 4 spurious cojump detection as we have 41233 observations for that pair. Now considering that the test may be somewhat oversized as the simulations show, we still find more cojumps than what is expected under the null. This demonstrates that these assets comove, to some extent, through cojumps. Because the S\&P 500 is most often negatively correlated, cojumps in the same direction are more readily identifiable: we detect 90 cojumps with same signs and 71 with opposite signs (deterministic jump simulation). This asymmetry is the multivariate equivalent of the difficulty of detecting small jumps on a highly volatile day. This asymmetry is also observed on figure 9 that reports the time series of the bootstrapped quantiles of the test statistic (that is, the quantiles for each intraday period).

The numbers of detected cojump with the bootstrap test (i.e. 161) is somewhat larger than those obtained as simultaneous univariate jumps (137). Moreover, we find that cojumps detected with both approaches are 56 (column "common coj."). This is entirely consistent with the simulation exercise that demonstrates conditions under which circumstances one or the other approach performs better.

Moreover, Table 5, column "coj. and j." shows the number of bootstrapped cojumps that were only flagged as jumps with the univariate approach. For the most conservative cojump test (line "with j"), we count 96 of these. This again confirms the intuition according to which price changes can be small relative to local variation, but still large relative to local covariation. Therefore, the univariate approach will detect large obvious cojumps but might miss less visible, but still abnormal, covariations.

Figure 10 reports the time series of detected bootstrapped cojump (i.e. the returns corresponding to a detected cojump). Figure 11, on the other hand, reports, for each day, the count of detected cojumps. We can observe on both figures an increase in cojump intensity in the middle of the sample which corresponds to the financial crisis. Moreover, the cojump size seems to increase during the financial turmoil (see Figure 10), that is, when news about bankruptcy, financial institutions acquisition, or government bailout plan regularly hit the market. To further identify the cojumps determinants for stock and bond returns, we would need to systematically match cojumps date and financial news. This exercise is beyond the scope of the paper but would be interesting for further research.

\subsubsection{Returns, volatility and correlation distribution}

What are the correlation and volatility conditions on days when cojumps are detected? Figures 15 provides the answer: we report the unconditional distribution of volatility and correlation VS the distribution of these measure conditional on a cojump detection. We cannot find any 
particularly distinguishing pattern for volatility and correlation on cojump days with respect to their unconditional distribution. This supports the idea that these cojumps are information driven, and not a mere artefact such as, for example, a trivial contrarian movement on a high correlation day. Figure 12, left panel, reports the count of cojumps per intra-day period. This allows to visualize if cojumps tend to be more likely at given times of the day. No sharp patterns are distinguishable, besides three spikes: at the opening ${ }^{7}$, at 12.45 and towards the end of the day. This also supports a link between macro news and cojumps (also supported in Lahaye, Laurent, and Neely (2011)), as these are arrival times of many scheduled news.

Finally, Figure 13 reports the distribution of returns for the S\&P and bond series, both unconditional and conditional on cojump occurrence. The latter conditional distribution tends to bi-bimodal and confirms that our approach detects small and large size co-jumps, in line in what we show in the Monte-Carlo exercise of Section 4.

\subsection{Exchange Rates and Bonds}

In this second empirical example, we analyze the jumps and cojumps for 30-year Treasury bonds (Bond) and the USD-JPY (JPY) exchange rate. The interest for bonds and currencies comovements has been revamped recently with the literature on carry trade and currency crashes. Carry trade is an investment strategy according to which an investor borrows money in a low interest rate currency to invest in another currency, usually in risk free government bonds likely to produce much higher returns. According to the uncovered interest rate parity (UIP) hypothesis the carry trade gains due to the interest-rate differential should be offset by a proportional depreciation of the investment currency. In a country suddenly increasing its interest rate and thereby attracting foreign capital, the UIP predicts an immediate appreciation of the currency. Empirical evidence however tend to support the opposite. Several attempts have been made recently to address the so-called "forward premium puzzle". The main explanation provided in the literature lies in the occurrence of currency crash due to market speculators.

As detailed in (Brunnermeier, Nagel, and Pedersen 2008), the depreciation process described by UIP is neutralized by speculators who hold on to their carry trade in the investment currency, as long as others do not unwind their positions, leading to a build up of an exchange rate bubble (Abreu and Brunnermeier 2003). When speculators suddenly unwind their carry trades, the price correction occurs as a currency crash. In what follows, we document the question of carry trade and currency crashes by analyzing the occurrence of jumps and cojumps between the JYP and the Bond.

For this second application, we use tick data from Olsen and Associates and Tick Data for the USD/JPY exchange rates and the 30-year U.S. T-bonds futures contracts series, respectively, over the period January 4, 2000 to January 10, 2013. Tick data are sampled at 15-minute frequency. As noted above, the 30-year Treasury bond are traded in the Chicago Board of Trade (CBOT) between 08:20-15:00 (EST). The exchange rate market on the other hand operates throughout the global 24-hour trading day. The overlapping period retained for the analysis is, therefore, 08:2015:00 (EST) on day $t$. Finally, the database is cleaned from days with too many missing values or low trading activity leaving 1865 days in the sample.

\footnotetext{
${ }^{7}$ The overnight return is ignored. The first return of the day is not overnight, but intraday.
} 


\subsubsection{Correlation}

Figure 5 (panel b) displays the daily correlations between the series. The estimates fluctuate widely over time, with most of the values ranging from -0.6 to 0.6. Contrasting with the SP500-Bond pair, it is not possible to identify long lasting periods over which correlations appear either positive or negative.

\subsubsection{Cojumps}

Columns 4-5 in Table 5 provide a detailed view on how the two pairs, Bond-JPY, jump. The univariate test allows to reject the null of no jumps in the JPY series for 1194 observations over 46878 , which produce a jump propensity of $2.55 \%$ per observation. The jump intensity is marginally smaller for the Bond series, with 1158 jumps detected over the same time period. Those findings emphasize the risk of large exchange rate movements, namely currency crashes, in the exchange rate market. Among those jumps only a few, namely 55, occur simultaneously.

We now turn to the bootstrap cojump tests. We start the analysis with the most conservative test which assumes a jump in each bootstrapped statistics. The number of detected cojumps is 81. It substantially exceeds the expected number of detections due to the type-I error: 4 for 46878 observations at 0.0001 level. Not bootstrapping jumps (normal-product case) leads to 206 cojumps. In line with the results on stock and bond returns, even the more conservative version of the test allows to detect more and different cojumps than the univariate approach stressing further the importance of considering covariations in cojump detection. The decomposition of the number of detected cojumps according to the sign of returns provides interesting features. Indeed, less than $20 \%$ of cojumps are associated with returns of the same sign. This suggests the presence of negative correlation in the tail, while the daily realized correlation measures fluctuate around zero. ${ }^{8}$

Unlike for the previous analyzed pair (S\&P 500 - bonds), we cannot observe different intensity across the sample period (see Figures 10 and 11). But mostly like the pair S\&P 500 - bonds, Figure 11 (displaying the count of cojumps per day) shows that most cojumps days exhibit one cojump, except a few days containing 2 or 3 cojumps. Moreover, some intra periods tend to exhibit more cojump than others (Figure 12): the first intraday return (which is not an overnight return), as well afternoon intra-periods. These patterns can be explained by scheduled news, at least partly.

\subsubsection{Returns, volatility and correlation distribution}

Figure 14 reports the distribution of returns for the JPY/USD and bonds series, both unconditional and conditional on cojump occurrence. As for stocks/bonds cojumps, we find rather bi-bimodal distributions for returns that are flagged as cojumps. The rather wide range of such cojump returns confirms the detection of both small and large size cojumps, in line with our simulations in Section 4.

Figure 16 reports the unconditional distribution of volatility and correlation VS the distribution of these measure conditional on a cojump detection. Here again, we do not find distinguishing pattern for volatility and correlation on cojump days with respect to their unconditional distribution.

\footnotetext{
${ }^{8}$ The symmetric volatility pattern around 0 is also reflected in the time series of the test quantiles on Figure 9.
} 


\subsection{Covariance Estimator Choice Robustness}

In our approach, we use the Brandt and Diebold (2006) no-arbitrage estimator for local covariance (see step 2 of the algorithm described in Section 4). This estimator has the virtue of parsimony: it reduces covariance estimation to a univariate problem and it is easily robustified against jumps (through individual assets' bipower variation) and cojumps (through the portfolio's bipower variation). How do result vary in function of the local covariance estimator? We expect some degree of sensibility to that choice. Indeed, different estimators have different degree of jump-robustness and therefore affect the cojump detection (this is analog to Corsi, Pirino, and Reno (2010) who detect more jumps using the threshold bipower variation).

We test two alternative estimators. First, we use realized volatility for local covariance estimation (see Figure 6), rather than bipower variation within the no-arbitrage approach of Brandt and Diebold (2006). Second, we use Boudt, Croux, and Laurent's (2011a) realized outlyingness weighted covariance (ROWCov).

As reported in Table 6, we find that RV detects considerably less cojumps in both pairs ( SP500-Bond and JPY-Bond). This highlights the importance of using robust estimations to detect abnormal comovements. Because realized volatility is less smooth than bipower variation, we detect less cojumps with realized volatility than with bipower variation (see Tables 5 and 6 ). Nevertheless, nothing prevents using other covariance estimators, not necessarily strictly based on a no-arbitrage argument as in Brandt and Diebold (2006).

We also test the sensitivity of our results using the realized outlyingness covariance of Boudt, Croux, and Laurent (2011a) as an alternative. ${ }^{9}$ The ROWCov (realized outlyingness weighted covariance) is affine equivariant and positive semidefinite (Boudt, Croux, and Laurent 2011a). Moreover, it is robust to successive jumps, unlike our approach using bipower variation in the Brandt and Diebold (2006) context. Therefore, we expect this measure to be smoother and allow to detect more cojumps. It is estimated in two steps. First, compute an outlyingness measure, similar to the statistic defined in Equation 3.3. That is, for a vector of returns $r_{t, j}$ :

$$
d_{t, j}=\frac{r_{t, j}^{\prime} \widehat{\Sigma_{t, j}^{-1}} r_{t, j}}{\Delta},
$$

where $\Delta$ is the length of an intraday interval. This initial step outlyingness measure requires a robust estimator $\widehat{\Sigma_{t, j}^{-1}}$. We follow Boudt, Croux, and Laurent (2011a) and choose the minimum covariance determinant (MCD) of Rousseeuw and van Driessen (1999).

Second, the covariance estimator is obtained with the weighted sum of the return's outer product:

$$
R O W \operatorname{Cov}_{t}=c_{w} \sum_{j=1}^{M} w\left(d_{t, j}\right) r_{t, j} r_{t, j}^{\prime}
$$

This estimator is a scaled ${ }^{10}$ realized covariance $\left(R \operatorname{Cov}_{t}=\sum_{j=1}^{M} r_{t, j} r_{t, j}^{\prime}\right)$ where returns contaminated by jumps/cojumps are down-weighted by the function $w$. In this paper, the retained weight-

\footnotetext{
${ }^{9}$ In this paper, we focus on these realized measures alternative. Whereas nothing prevents the use of a parametric approach such as a dynamic conditional correlation model, we keep our focus on realized estimators. This allows to remain consistent in terms of high-frequency data exploitation throughout our method, and get a measure which is sensitive to small daily variation, as opposed to a smoother, more persistent parametric estimator.

${ }^{10}$ The scaling constant $c_{w}$ ensures that the ROWCov estimator is consistent for the integrated covariance under a Brownian semimartingale with jumps (Eq. 2.1). It depends on the process dimension (2 in our case), as well as
} 
ing function is the so-called soft-rejection:

$$
w(z)=\min 1, k / z,
$$

where $k$ is a tuning parameter set to the $\chi^{2}$ distribution $\beta$ quantile. For this application, we choose $\beta=0.95$.

Results using this estimator are reported in Table 7. We find significantly more bootstrapped cojumps with this approach on the Bond-JPY pair (148 in the conservative bootstrap case simulating jumps at each observation VS 81 in the approach using Brandt and Diebold (2006) with bipower variation). On the other pair, S\&P 500 - Bonds, the detected jumps with ROWCov is slightly lower: 146 vs. 161. This result, as expected, shows how cojump detection can vary in function of the robustness of the covariance estimator. For example, the ROWCov estimator is robust to successive jumps, unlike the bipower variation approach within the Brandt and Diebold (2006) estimator, leading to differences in cojump detection.

\section{Conclusion}

In this paper, we study the question as to whether S\&P 500, US bonds and currencies tend to comove together through cojumps, beyond the mere simultaneous occurrence of large jumps. We propose a simple approach to test for such movements: generating the distribution of the returns' product at any intraday period through a parametric bootstrap, under different null hypothesis scenarios.

We show that our approach provides good results in terms of size and power. This holds also true relative to the existing literature. Moreover, this approach is simple to implement and it is flexible.

When applied to market-wide assets, we often reject null hypothesis of no-cojumps, much more often than the chosen nominal sizes, as well as empirical sizes obtained from the Monte Carlo evaluation of Section 4. This supports the idea that the total covariation is partly attributable to cojumps, and not only large simultaneous jumps.

The application of our cojumps test to real data - S\&P 500, US bonds and currencies - provides interesting features. We detect more and different cojumps for the S\&P 500 - US bonds pair as well as the US bond - USD-JPY exchange rate pair with the bootstrap procedure with respect to the the univariate approach based on the modified Lee-Mykland test. Second, while the empirical literature relying on parametric and nonparametric measures of correlation tends to support the existence of a negative correlation between stocks and bonds, the majority of cojumps detected occur for returns with the same sign suggesting the existence of a positive correlations between the two types of assets in the tails. Third, the cojumps intensity is relatively stable over time but increases during the crisis period. The cojumps detection for the second application on US bonds and the USD-JPY exchange rate confirms that our test allows to detect much more and different cojumps than the univariate approach.

Cojumps are of interest as they may contain special information content that can be useful to understand dynamics and potentially improve volatility-correlation modeling-prediction. Cojumps

the weight function $w$. See (Boudt, Croux, and Laurent 2011a) for full details, where the constant value is provided for different combination of system dimension and quantile $\beta$. 
are also important for risk management and option pricing. Applications of interest are also within the contagion literature, as well as systemic risk assessment. For the latter purpose, it is of interest to understand how an institution is correlated with the system as a whole, notably through cojumps. Hence the interest of a cojump test that work with $n$ assets, even for small $n$. All these questions are under current investigation as subsequent research. 


\section{References}

Abreu, D., and M. Brunnermeier (2003): "Bubbles and Crashes," Econometrica, 71, 173-204.

Aїт-Sahalia, Y. (2004): "Disentengling diffusion from jumps," Journal of Financial Economics, $74,487-528$.

AÏT-Sahalia, Y., and J. Jacod (2006): "Testing for Jumps in a Discretely Observed Process," Forthcoming in Annals of Statistics.

Andersen, T., T. Bollerslev, and D. Dobrev (2007): "No-arbitrage Semi-martingale Restrictions for Continous-time Volatility Models Subject to Leverage Effects, Jumps and i.i.d. noise: Theory and Testable Distributional Implications," Journal of Econometrics, 138, 125-180.

Andersen, T. G., D. Dobrev, and E. Schaumburg (2008): "Jump Robust Volatility Estimation," Mimeo.

Aroian, L. A. (1944): "The probability function of the product of two normally distributed variables," The Anals of Mathematical Statistics, 18, 265-271.

Aslanidis, N., And C. Christiansen (2010): "Coherent measures of risk," Mathematical Finance, 9, 203-228.

Baele, L., B. G., and K. Inghelbrecht (2010): "The Determinants of Stock and Bond Return Comovements," Review of Financial Studies, 23, 2374-2428.

Barndorff-Nielsen, O., And N. Shephard (2004): "Power and bipower variation with stochastic volatility and jumps (with discussion)," Journal of Financial Econometrics, 2, 1-37.

(2006): "Econometrics of testing for jumps in financial economics using bipower variation," Journal of Financial Econometrics, 4, 1-30.

Baur, D., And B. Lucey (2009): "Flight-to-quality or Contagion? An Empirical Analysis of Stock-bond correlations," Journal of Financial Stability, 5, 339-352.

Boffelli, S., J. Novotny, and G. Urga (2014): "A Frequency-Specific Factorization to Identify Commonalities with an Application to the European Bond Markets," Mimeo.

Bollerslev, T., T. H. Law, and G. Tauchen (2008): "Risk, Jumps, and Diversification," Journal of Econometrics, 144, 234-256.

Boudt, K., C. Croux, and S. Laurent (2011a): "Outlyingness Weighted Covariation," Journal of Financial Econometrics, 9, 657-684.

(2011b): "Robust estimation of intraweek periodicity in volatility and jump detection," Journal of Empirical Finance, 18, 353-367.

Brandt, M. W., and F. X. Diebold (2006): "A no arbitrage approach to range based estimation of return covariances and correlations," Journal of Business, 79, 61-74.

Brunnermeier, M. K., S. Nagel, and L. H. Pedersen (2008): "Carry Trades and Currency Crashes," NBER Working Paper 14473. 
Corsi, F., D. Pirino, and R. Reno (2010): "Threshold bipower variation and and the impact of jumps on volatility forecasting," Journal of Econometrics, 159, 276-288.

Craig, C. C. (1936): "On the frequency function of xy," The Annals of Mathematical Statistics, $7,1-15$

Dumitru, A. M., and G. Urga (2012): "Identifying jumps in financial assets: a comparison between non-parametric jump tests," Forthcoming in Journal of Business and Economic Statistics.

EnGLE, R. (2002): "Dynamic conditional correlation: A simple class of multivariate generalized autoregressive conditional heteroskedasticity models," Journal of Business and Economic Statistics, 20, 339-350.

Engle, R. F., And S. Kozicki (1993): "Testing for common features," Journal of Business and Economic Statistics, 11, 369-380.

Gobbi, F., and C. Mancini (2012): "Identifying the Brownian Covariation From the Co-jumps given discrete observations," Econometric theory, 28, 249-273.

Gordon, N. J., D. J. Salmond, And A. F. M. Smith (1993): "Novel Approach to Nonlinear/non-Gaussian Bayesian State Estimation," IEE Proceedings-F, 140, 107-113.

Guidolin, M., And A. Timmermann (2006): "Dynamics in the Joint Distribution of Stock and Bond Returns," Journal of Applied Econometrics, 21, 1-22.

JACOD, J., AND V. TODOROV (2009): "Testing for common arrival of jumps in discretely-observed multidimensional processes," Annals of Statistics, 37, 1792-1938.

Jiang, G. J., and R. C. A. Oomen (2008): "Testing for Jumps When Asset Prices are Observed with Noise: A "Swap Variance" Approach," Journal of Econometrics, 144, 352-370.

Jondeau, E., J. Lahaye, and M. Rockinger (2013): "Jump detection in a high-frequency dynamic microstructure model: a particle filter estimation," Mimeo.

Ju, C. (2010): "Testing for Jumps and Cojumps in Financial Markets," Thesis Submitted to the School of Engineering in partial fulfillment of the requirements for the degree ofMaster of Science in Computation for Design and Optimization, MIT, Boston.

Lahaye, J., S. Laurent, and C. J. Neely (2011): "Jumps, cojumps and macro announcements," Journal of Applied Econometrics, 26, 893-921.

Lee, S. S., and P. A. Mykland (2008): "Jumps in Financial Markets: A New Nonparametric Test and Jump Dynamics," Review of Financial Studies, doi: 10.1093/rfs/hhm056.

Mancini, C. (2009): "Non-parametric threshold estimation for models with stochastic diffusion coefficient and jumps," Scandinavian Journal of Statistics, 36, 270-296.

PodolskiJ, M., And D. Ziggel (2010): "New Tests for Jumps in Semimartingale Models," Statistical Inference for Stochastic Processes, 13, 15-41. 
Rousseeuw, P., and K. van Driessen (1999): "A fast algorithm for the minimum covariance determinant estimator," Technometrics, 41, 212-223.

Ware, R., And F. LAD (2003): "Approximating the Distribution for Sums of Products of Normal Variables," Department of Mathematics and Statistics, University of Canterbury, New Zealand, Report UCDMS 2003/15.

YANG, J., Y. ZHOU, AND Z. WANG (2009): "The stock-bond correlation and macroeconomic conditions: One and a half centuries of evidence," Journal of Banking E Finance, 33(4), 670680 . 
Figure 1: One simulated day of trading: prices $p_{t_{j}}^{* i}$

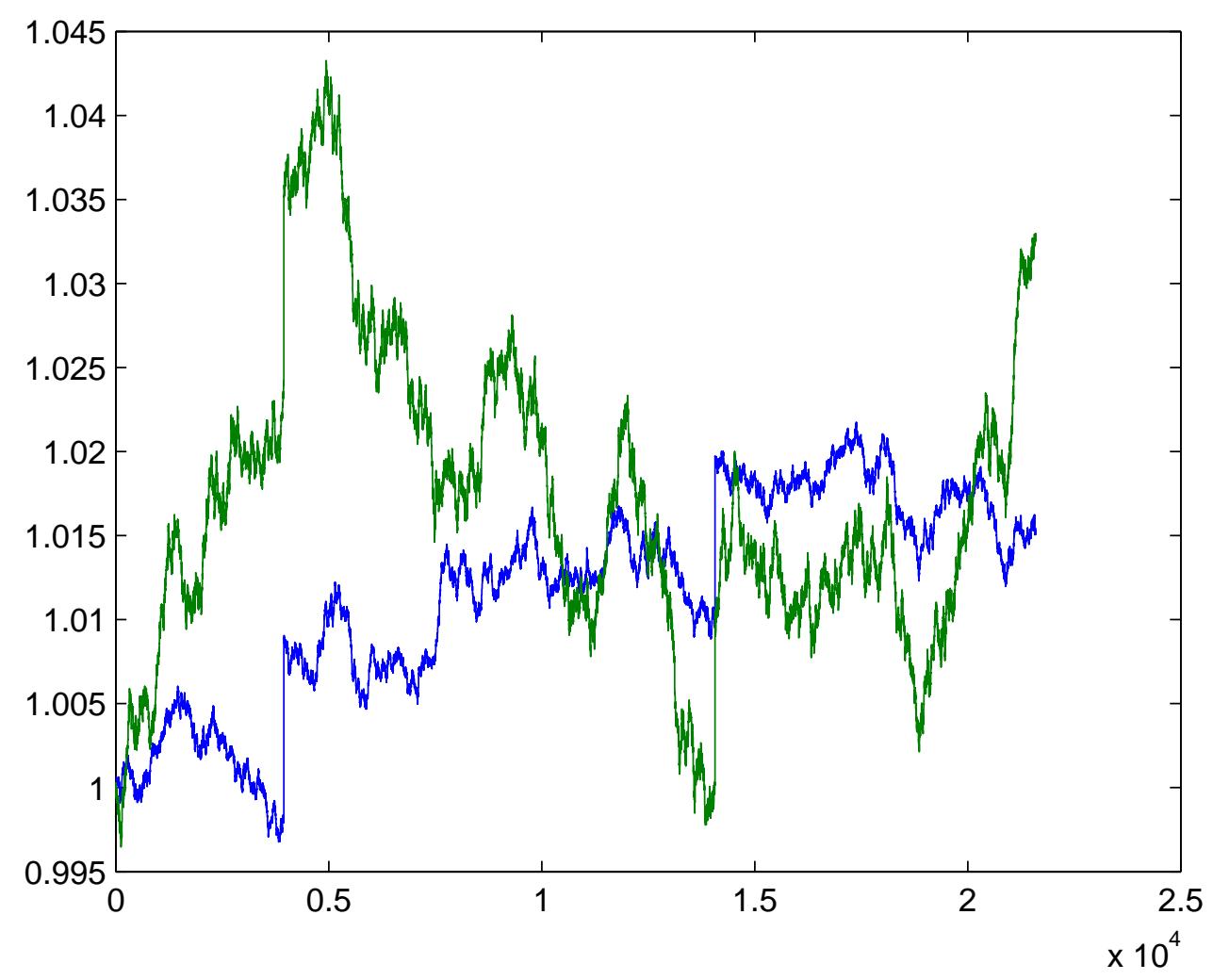


Figure 2: One simulated day of trading: prices $p_{t_{j}}^{* i}$ sampled every 5 minutes

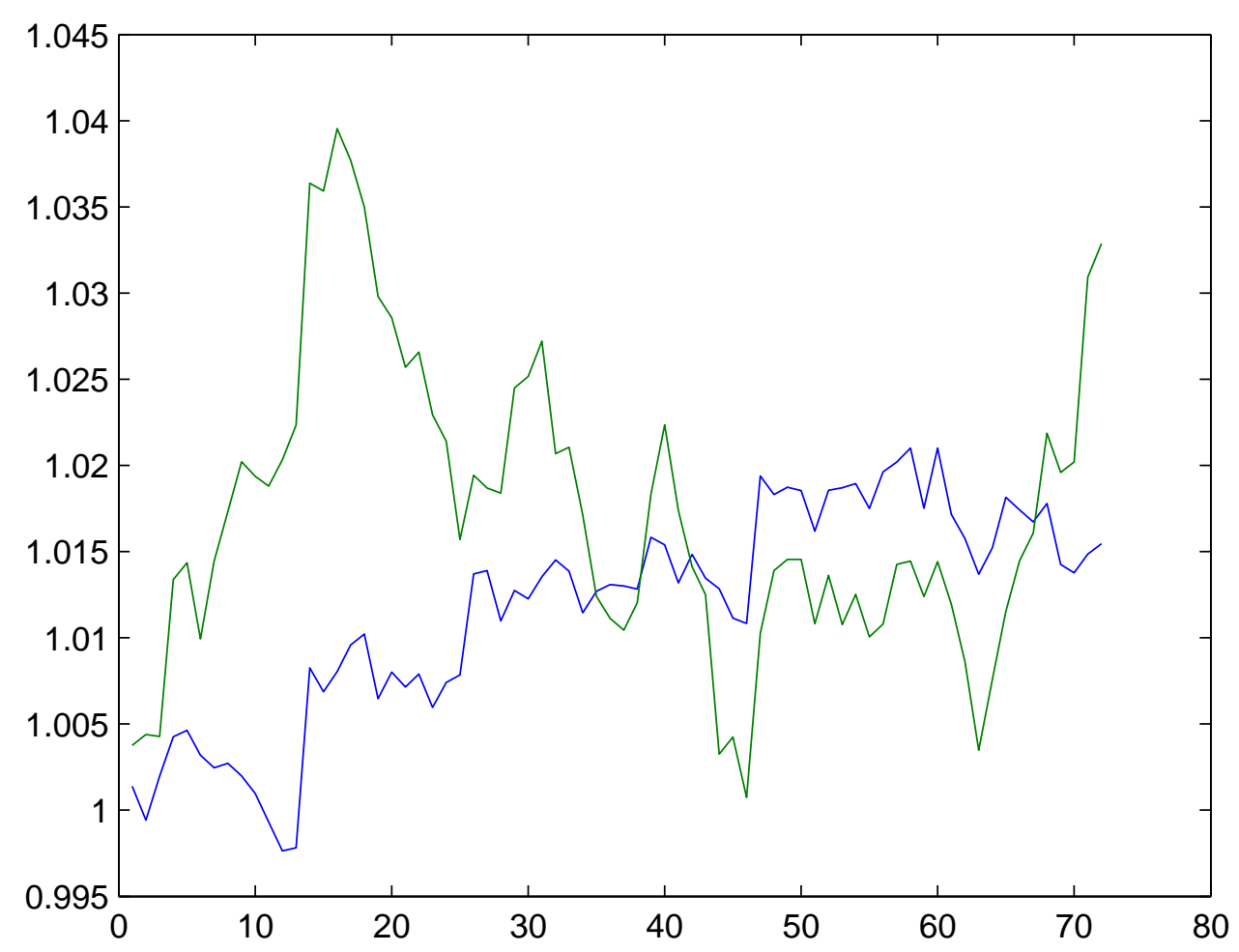


Table 1: Simulation calibration

\begin{tabular}{|c|c|c|}
\hline Variable name & Description & Value \\
\hline$K$ & seconds per day & $6^{*} 60 * 60=21600$ \\
\hline cDays & count of days & 100 \\
\hline$\sigma_{1}$ & asset 1 volatility & $\{0.0001 ; 0.0002\}$ \\
\hline$\sigma_{2}$ & asset 2 volatility & 0.0001 \\
\hline$\rho$ & Brownian increments correlation & $\{ \pm 0.1 ; \pm 0.3 ; \pm 0.5 ; \pm 0.7 ; \pm 0.9\}$ \\
\hline$\Delta$ & sampling frequency in minutes & 5 \\
\hline \multicolumn{3}{|c|}{ Scenario 1} \\
\hline$\lambda$ & cojump intensity & 3 \\
\hline$J_{s i z e}$ & cojump size & $\{0.004 ; 0.005 ; 0.01\}$ \\
\hline$\lambda_{1}$ & asset 1 jump intensity & 0 \\
\hline$J_{s i z e, 1}$ & asset 1 jump size & - \\
\hline$\lambda_{2}$ & asset 2 jump intensity & 0 \\
\hline$J_{\text {size }, 2}$ & asset 2 jump size & - \\
\hline \multicolumn{3}{|c|}{ Scenario 2} \\
\hline$\lambda$ & cojump intensity & 0 \\
\hline$J_{\text {size }}$ & cojump size & - \\
\hline$\lambda_{1}$ & asset 1 jump intensity & 3 \\
\hline$J_{s i z e, 1}$ & asset 1 jump size & $\{0.004 ; 0.005 ; 0.01\}$ \\
\hline$\lambda_{2}$ & asset 2 jump intensity & 0 \\
\hline$J_{\text {size }, 2}$ & asset 2 jump size & - \\
\hline \multicolumn{3}{|c|}{ Scenario 3} \\
\hline$\lambda$ & cojump intensity & 0 \\
\hline$J_{\text {size }}$ & cojump size & - \\
\hline$\lambda_{1}$ & asset 1 jump intensity & 0 \\
\hline$J_{\text {size }, 1}$ & asset 1 jump size & - \\
\hline$\lambda_{2}$ & asset 2 jump intensity & 0 \\
\hline$J_{\text {size }, 2}$ & asset 2 jump size & - \\
\hline
\end{tabular}




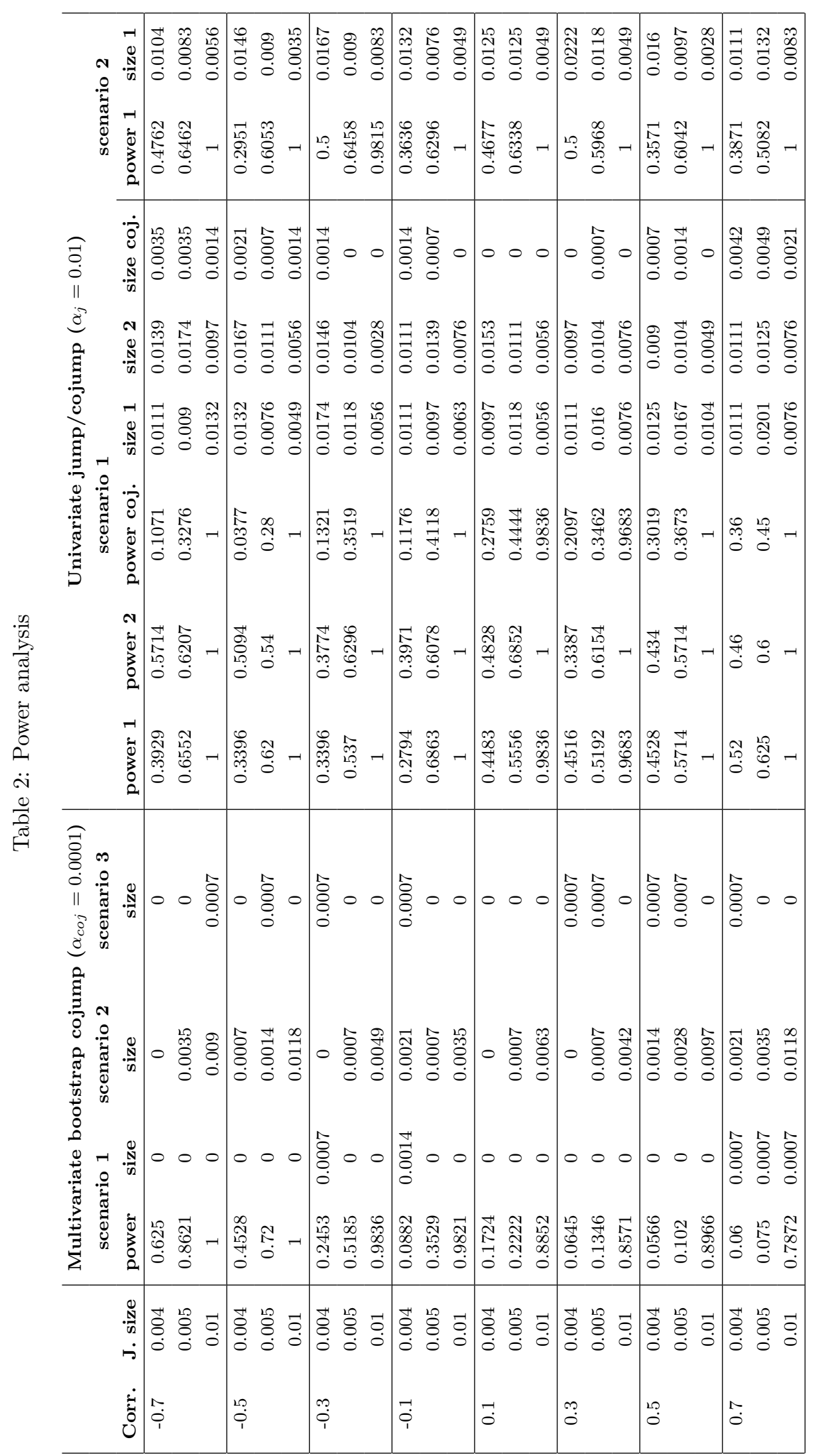

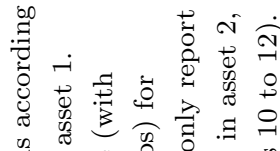

.

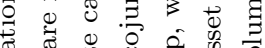

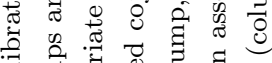

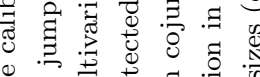

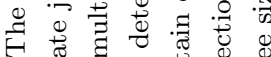

कि

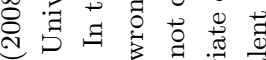

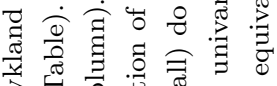

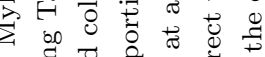

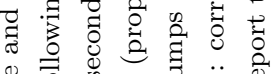

\&

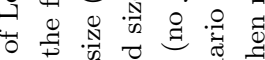

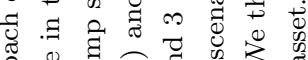

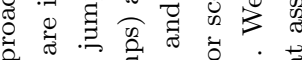

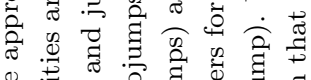

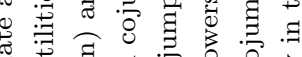

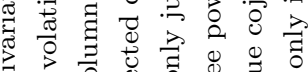

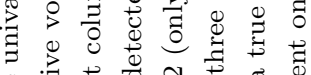

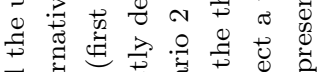

䒕

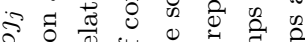

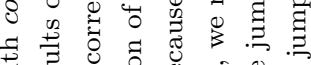

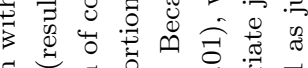

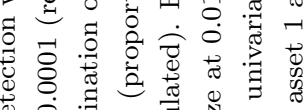

일

द्वे

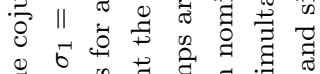

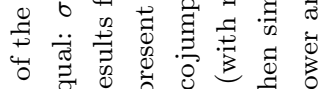

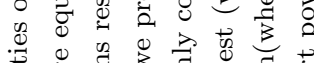

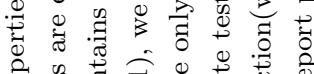

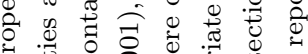

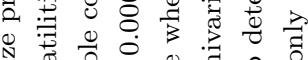

娄

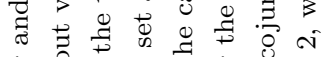

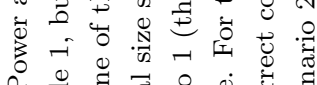

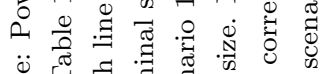

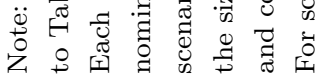




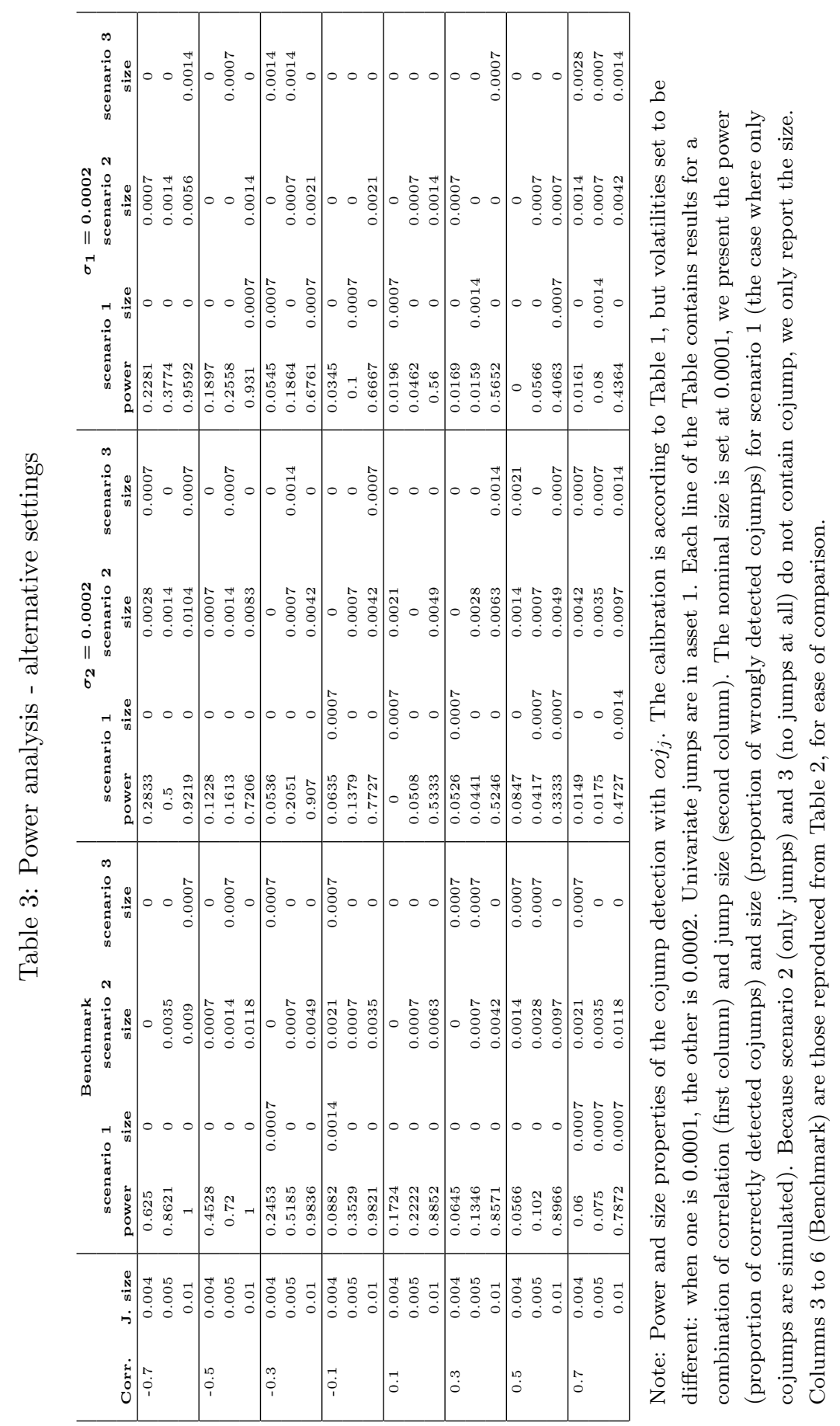


Table 4: Description of original series

\begin{tabular}{|c|c|c|c|c|}
\hline Asset & Source & Freq & Trading Hours (EST) & Period available \\
\hline \hline S\&P 500 Futures (SP) & CME & tick & $9.30-16.15$ & $04 / 01 / 2005-10 / 01 / 2013$ \\
\hline 30-year US Treasury bond (US) & CBOT & tick & $8.20-15.00$ & $04 / 01 / 2005-10 / 01 / 2013$ \\
\hline USD/JPY & O\&A & tick & 24 hours a day & $04 / 01 / 2005-10 / 01 / 2013$ \\
\hline
\end{tabular}




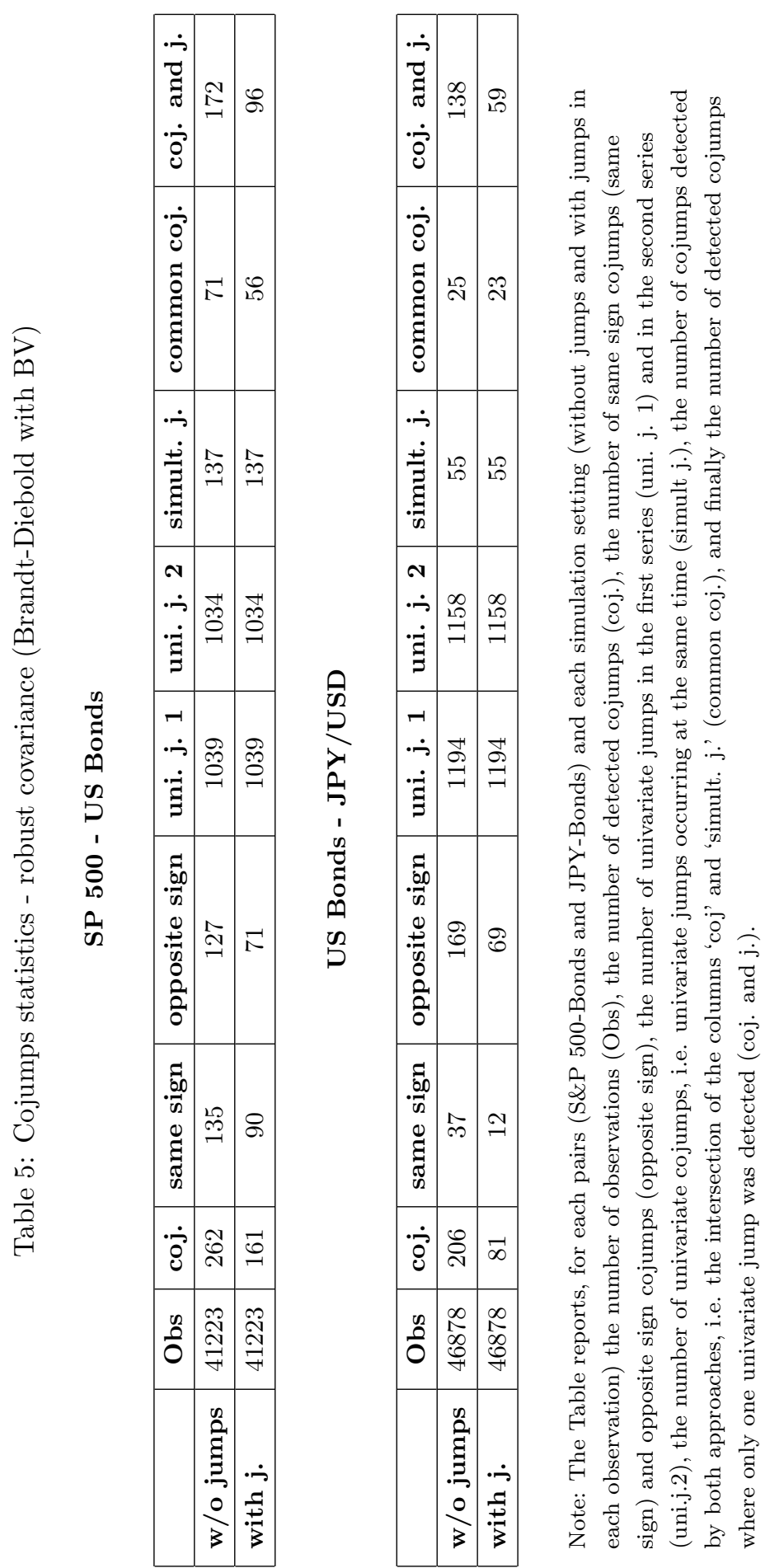




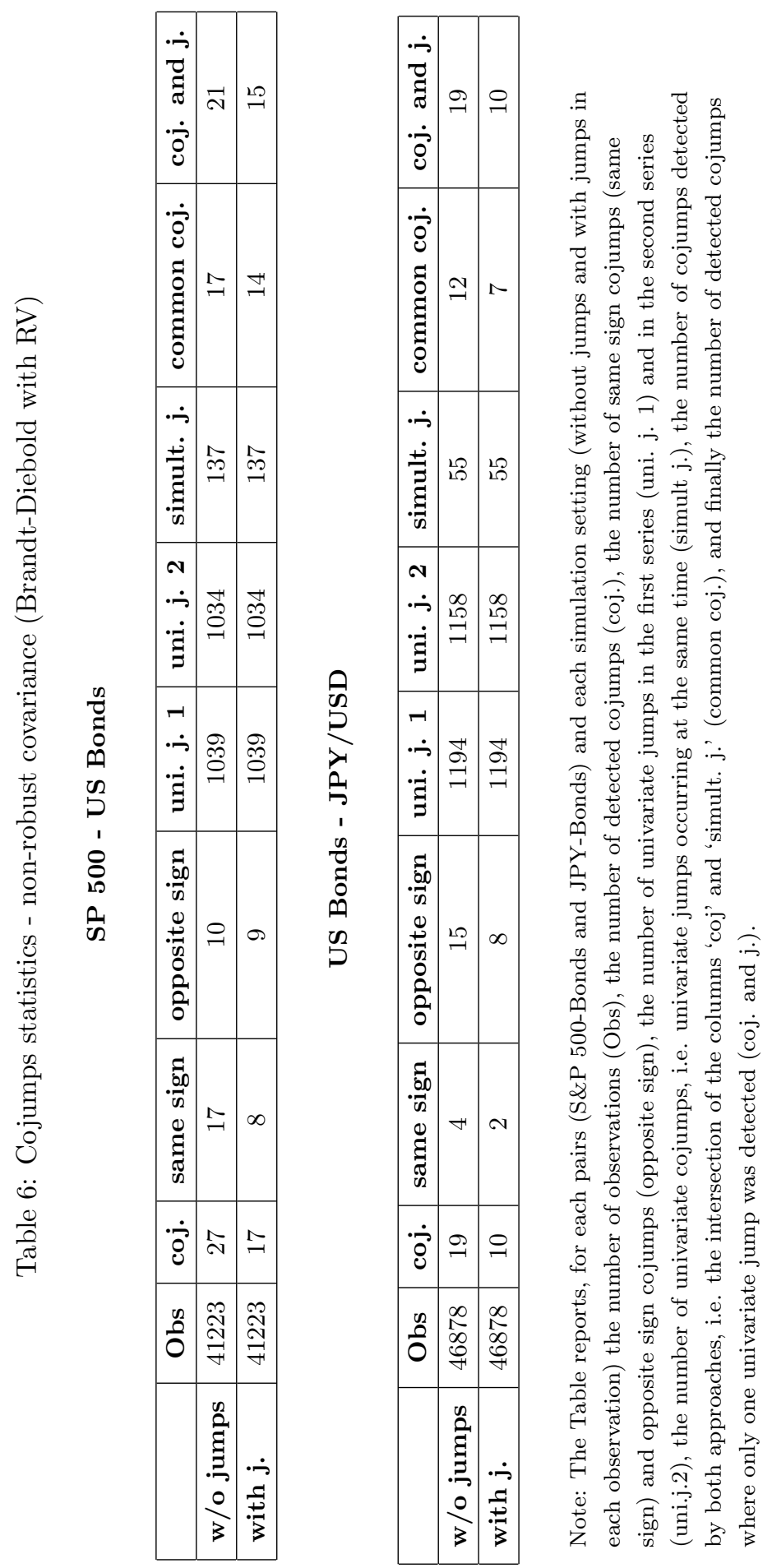




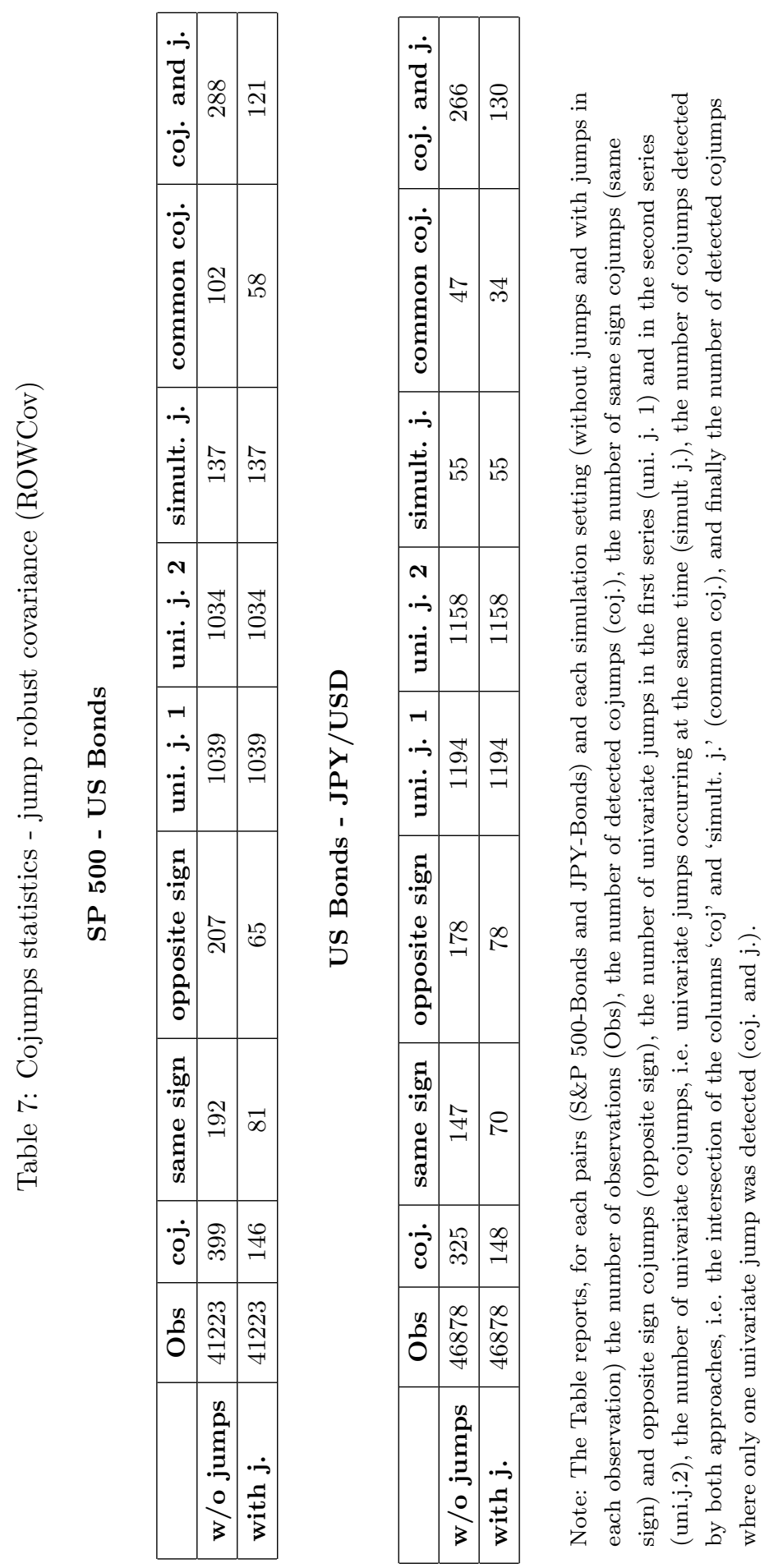




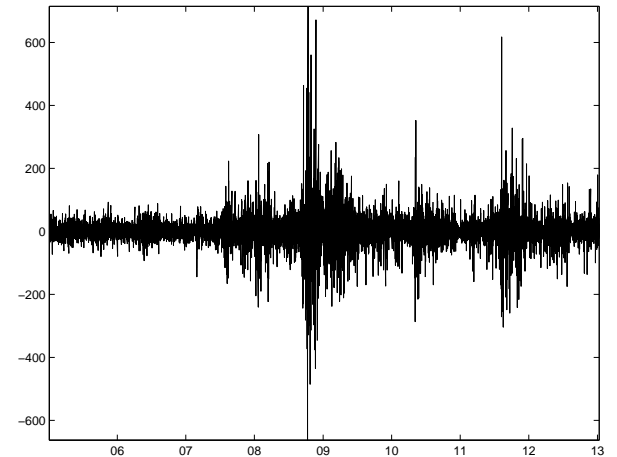

(a) S\&P 500

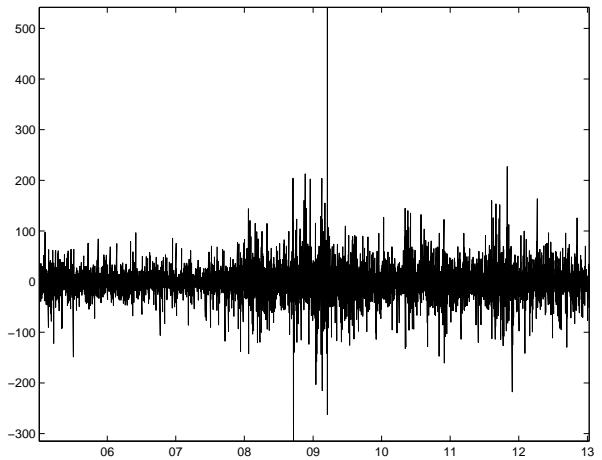

(b) US bonds

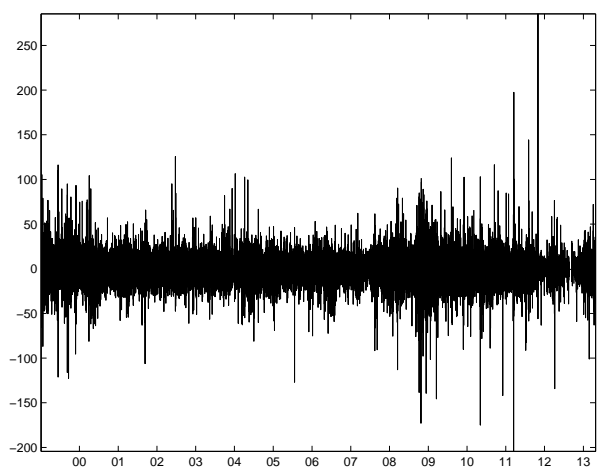

(c) JPY/USD

Figure 3: Intraday 15-minute returns in basis points (log-price difference $\times 10000)$ for the S\&P 500, US bonds, and JPY/USD 


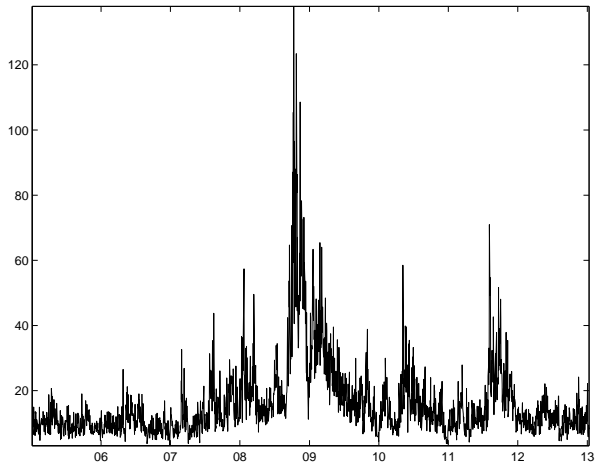

(a) S\&P 500

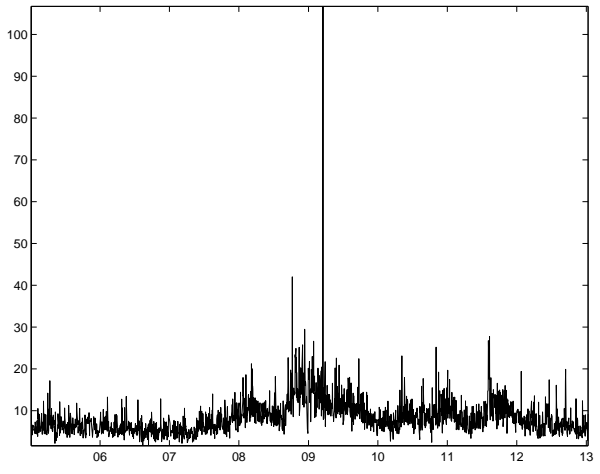

(b) US bonds

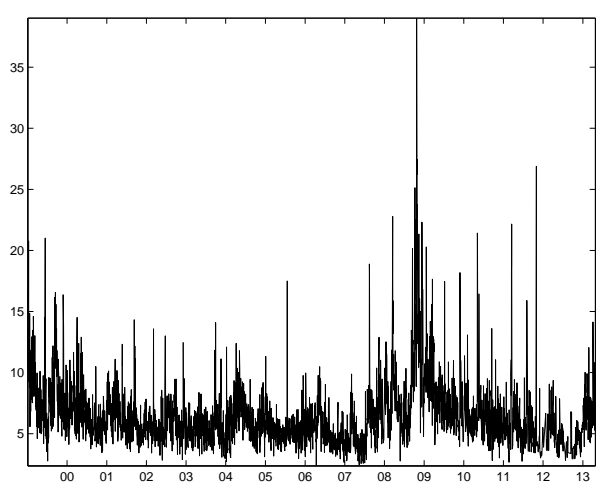

(c) JPY/USD

Figure 4: Daily realized volatility (scaled per intraday (15-minute) interval): S\&P 500, US bonds, JPY/USD 


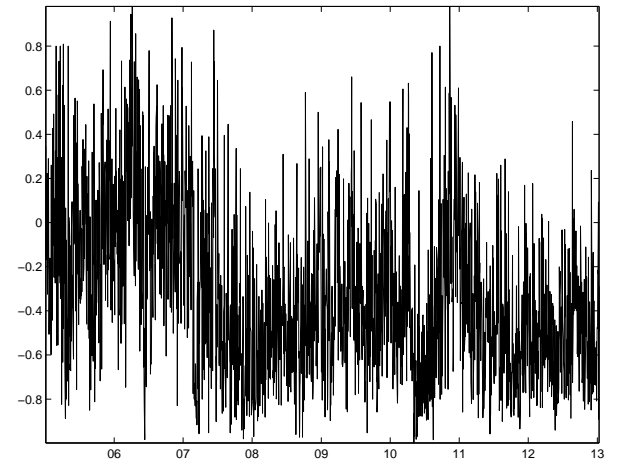

(a) S\&P 500-Bonds

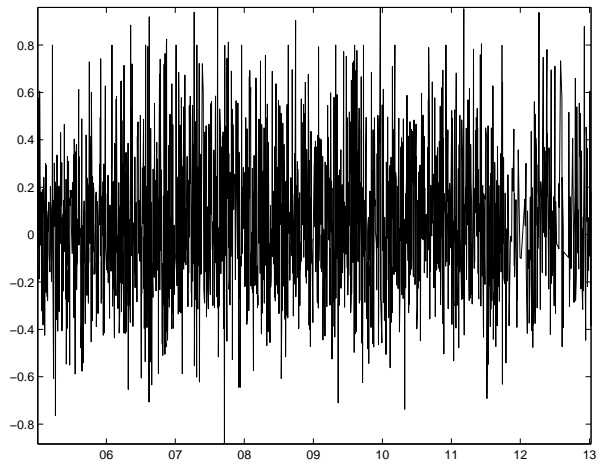

(b) Bonds-JPY/USD

Figure 5: No-arbitrage daily realized correlation (using bi-power variation) - S\&P 500-Bonds and Bonds-JPY/USD

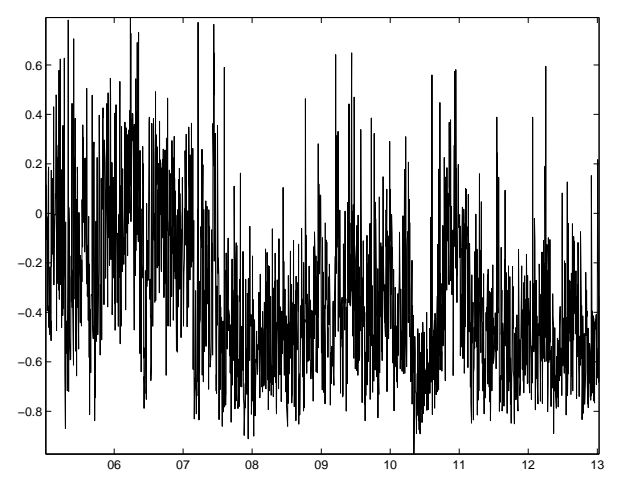

(a) S\&P 500-Bonds

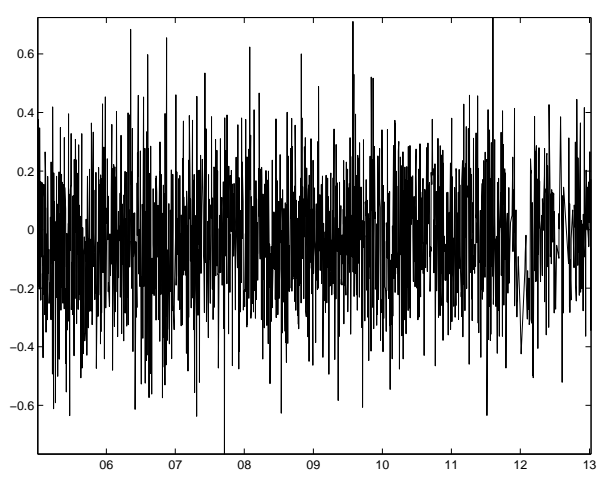

(b) Bonds-JPY/USD

Figure 6: No-arbitrage daily realized correlation (using realized volatility) - S\&P 500-Bonds and Bonds-JPY/USD 


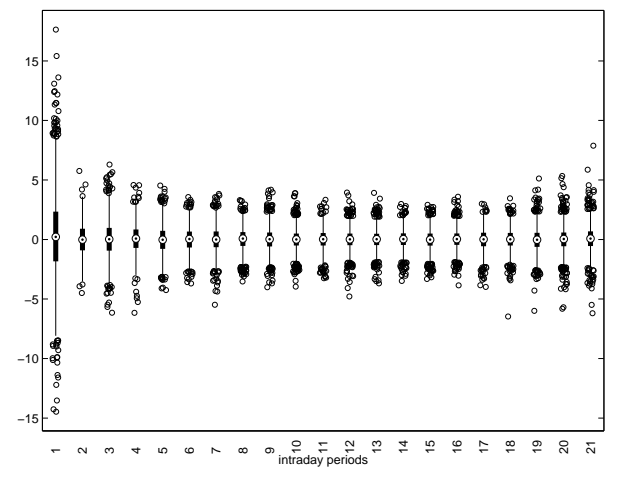

(a) S\&P 500

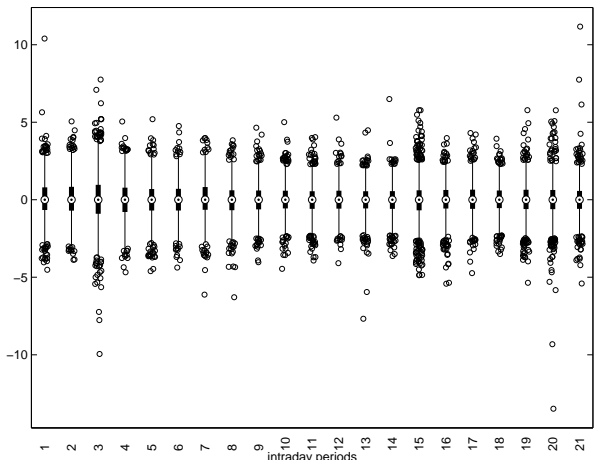

(b) US bonds

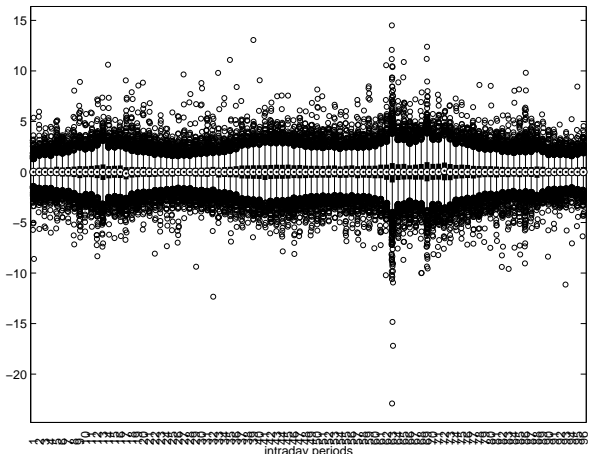

(c) JPY/USD

Figure 7: Intraday periodicity. Boxplot of returns conditional on their intraday period - S\&P 500, US bonds, and JPY/USD. Returns are previously standardized by their corresponding scaled daily volatility (estimated with bipower variation). 


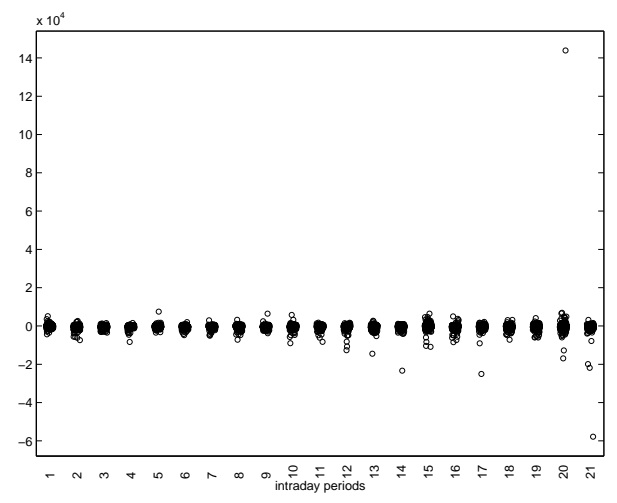

(a) S\&P500-Bonds

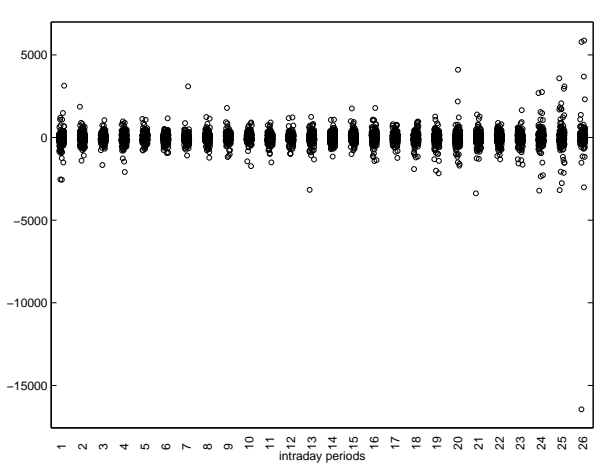

(b) Bonds-JPY/USD

Figure 8: Intraday coperiodicity. Boxplot of returns's product conditional on their intraday period - S\&P 500-Bonds and Bonds-JPY/USD. Returns are previously corrected for intraday periodicity. The product is therefore computed on periodicity free returns. 


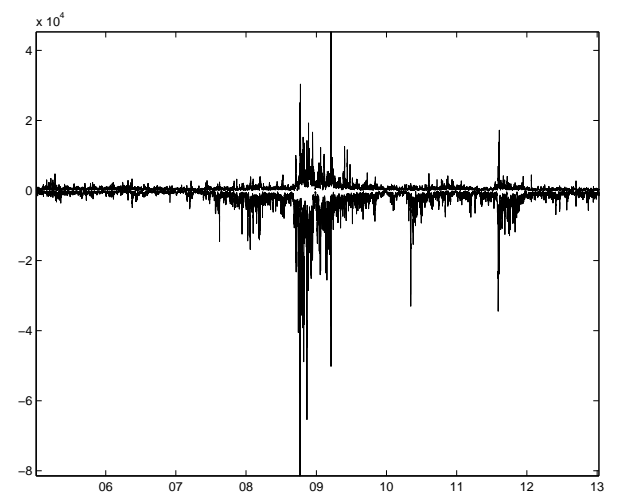

(a) S\&P 500-Bonds

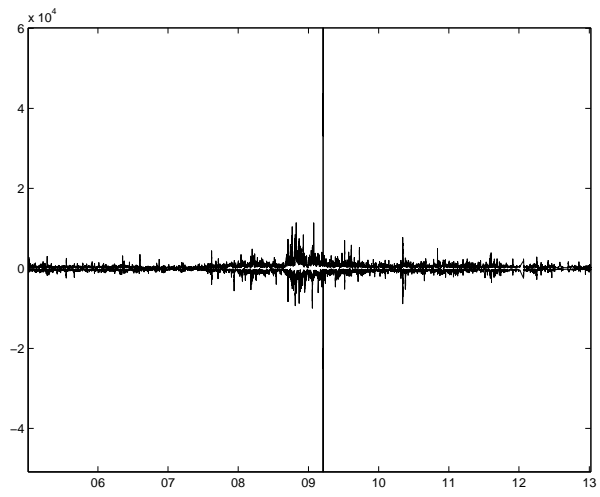

(b) Bonds-JPY/USD

Figure 9: Time varying intraday quantiles of $c o j_{t}$ under the null of deterministic idiosyncratic jumps (with size equal to the maximum intraday return). S\&P 500-Bonds and Bonds-JPY/USD 

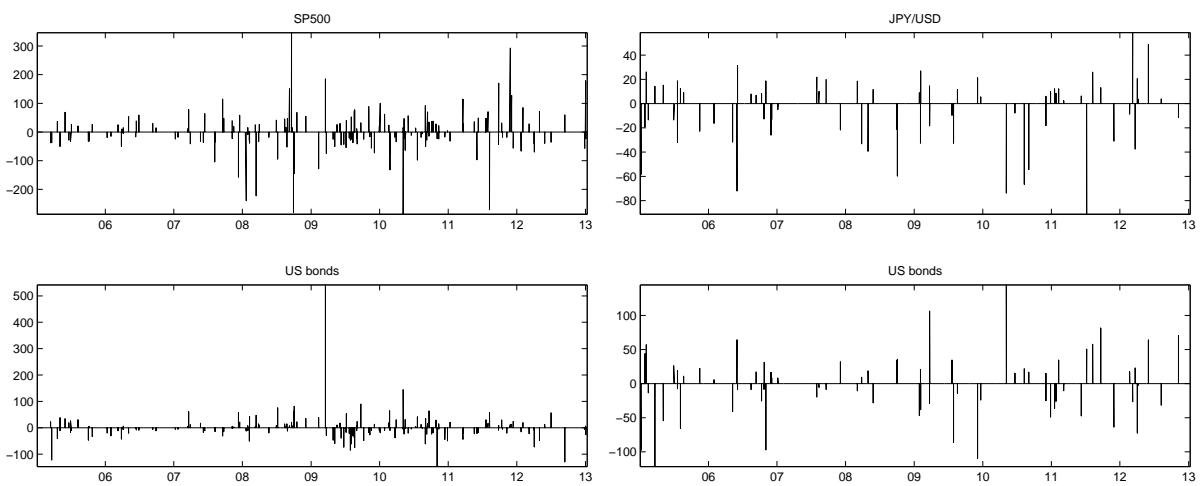

(a) S\&P 500/Bonds

(b) Bonds-JPY/USD

Figure 10: Detected bootsrapped cojumps time series: S\&P 500-Bonds and Bonds-JPY/USD 


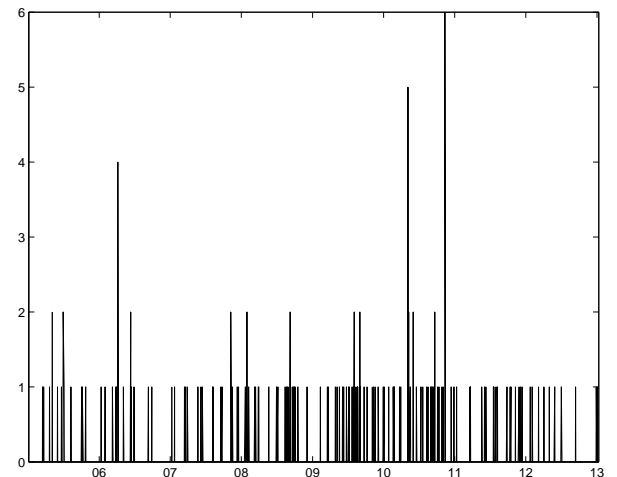

(a) S\&P 500-Bonds

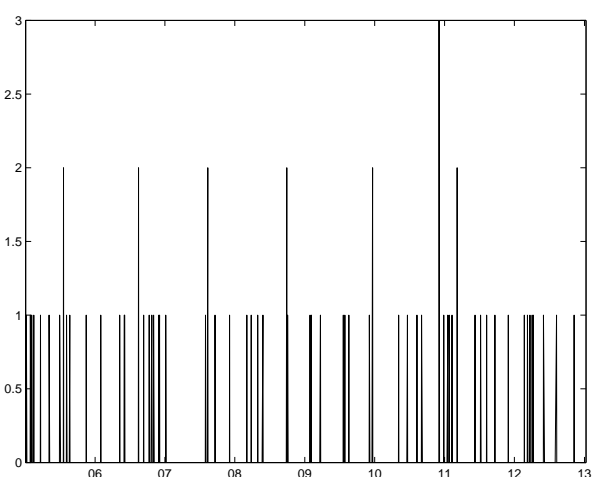

(b) Bonds-JPY/USD

Figure 11: Count of cojumps per day- S\&P 500-Bonds and Bonds-JPY/USD 


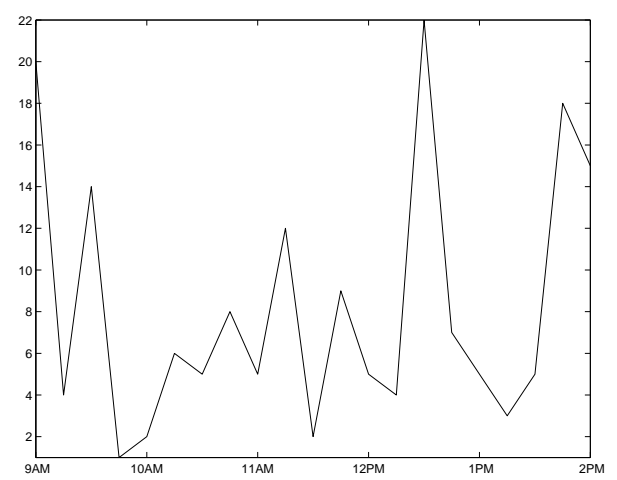

(a) S\&P 500-Bonds

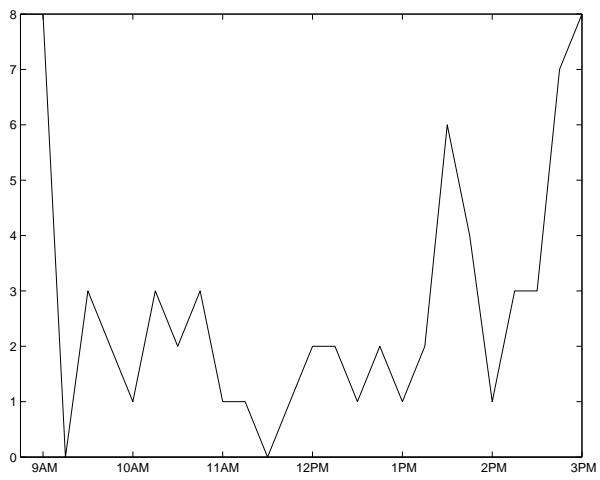

(b) Bonds-JPY/USD

Figure 12: Count of cojumps per intraday period- S\&P 500-Bonds, Bonds-JPY/USD 


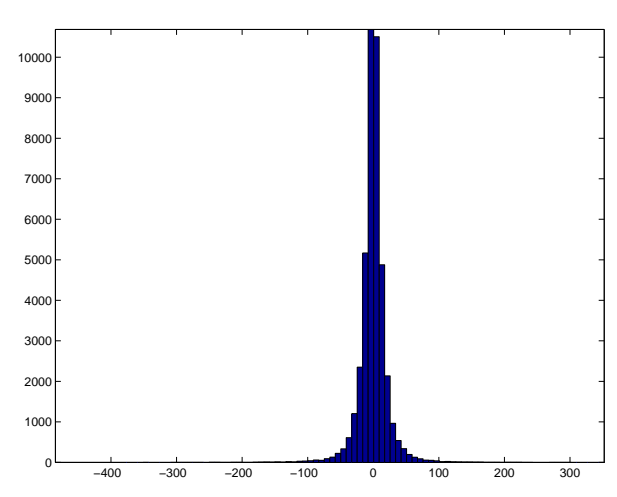

(a) S\&P 500 returns

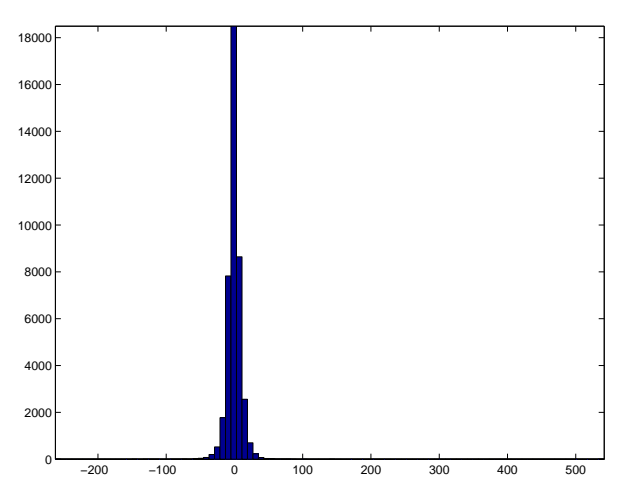

(c) Bond returns

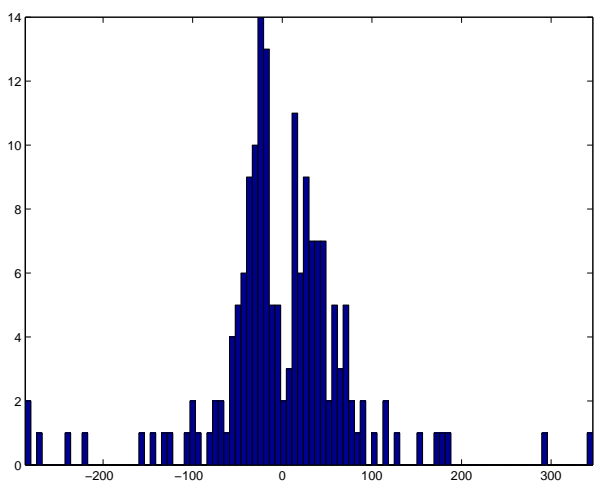

(b) S\&P 500 - cojump returns

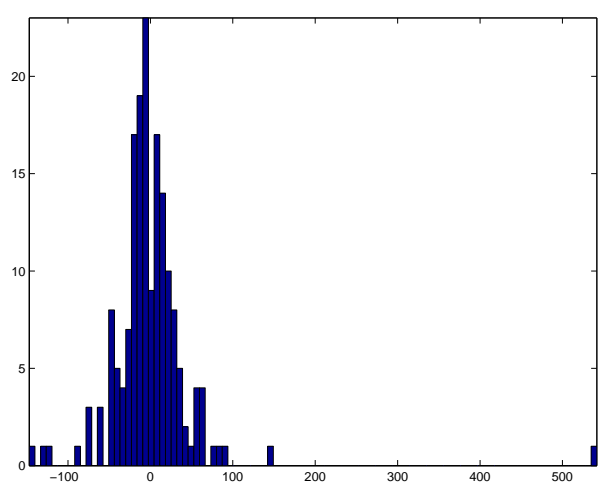

(d) Bond - cojump returns

Figure 13: S\&P 500 and US bonds intraday returns distributions: unconditional vs. cojump returns 


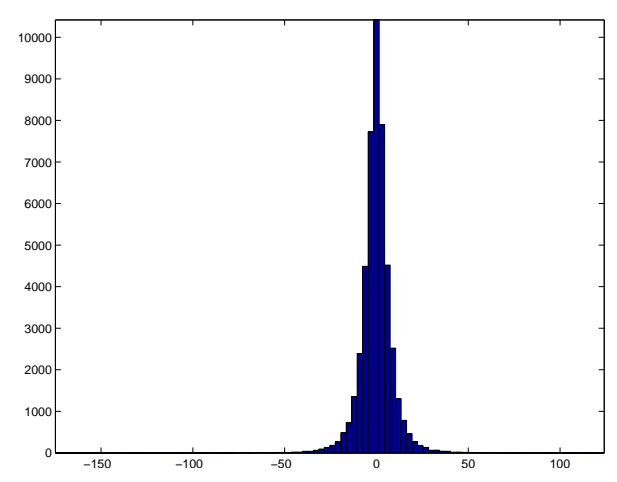

(a) JPY/USD returns

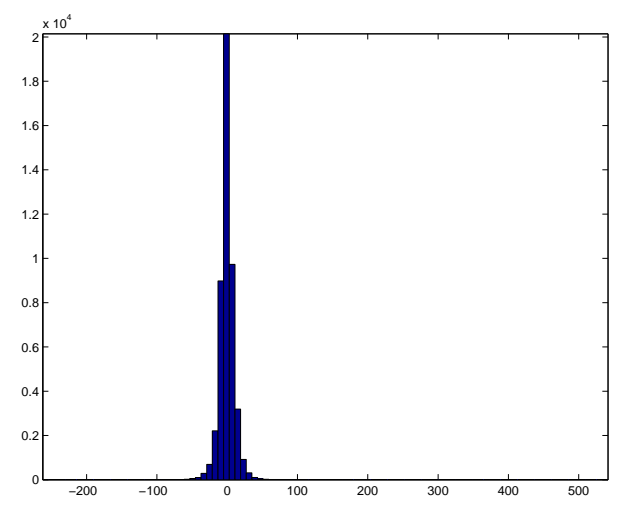

(c) Bond returns

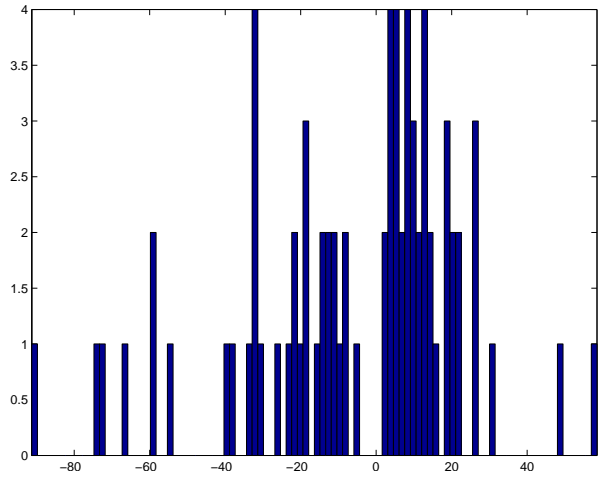

(b) JPY/USD - cojump returns

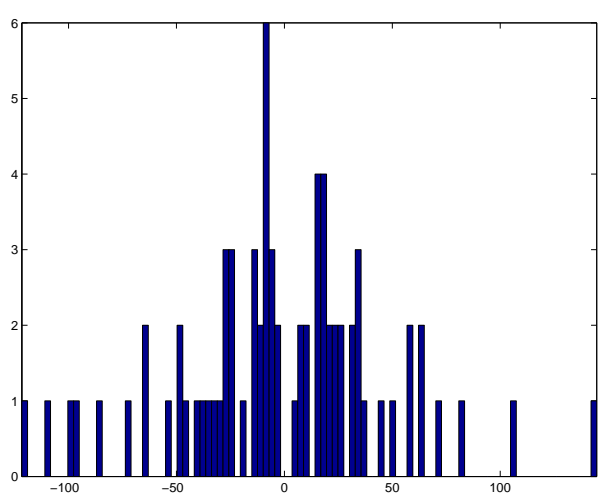

(d) Bond - cojump returns

Figure 14: JPY/USD and US bonds intraday returns distributions: unconditional vs. cojump returns 


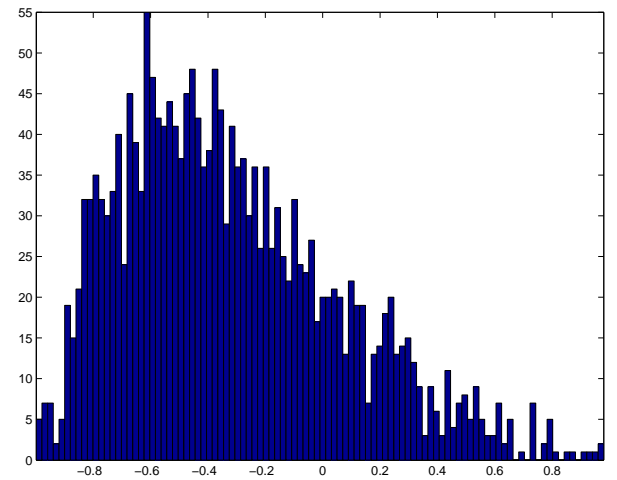

(a) correlation

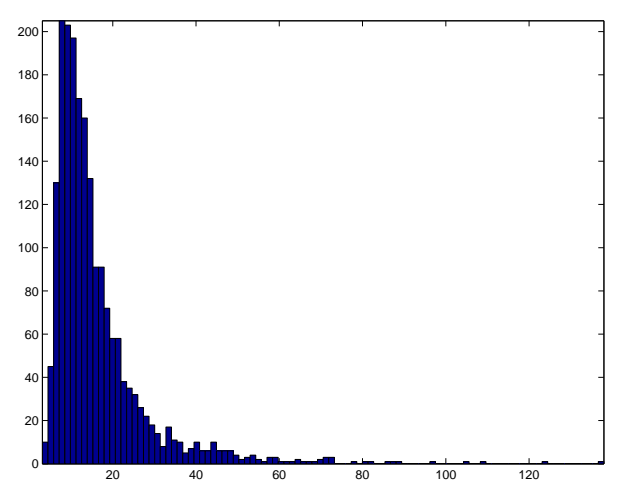

(c) volatility $\mathrm{S} \& \mathrm{P} 500$

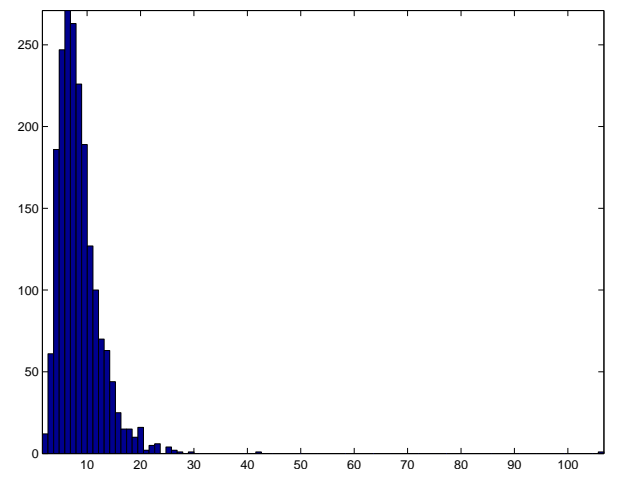

(e) volatility bonds

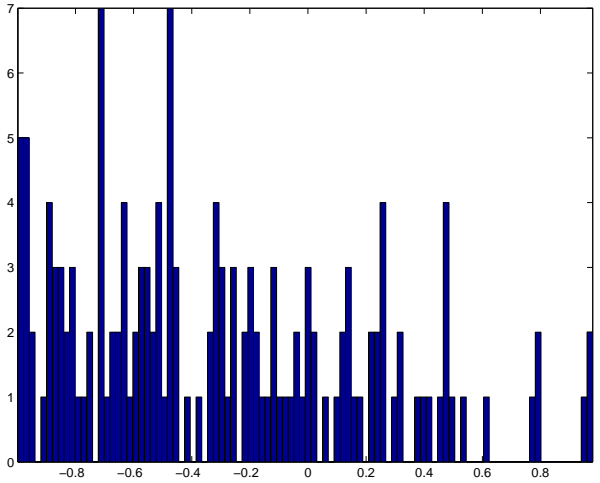

(b) correlation - cojump days

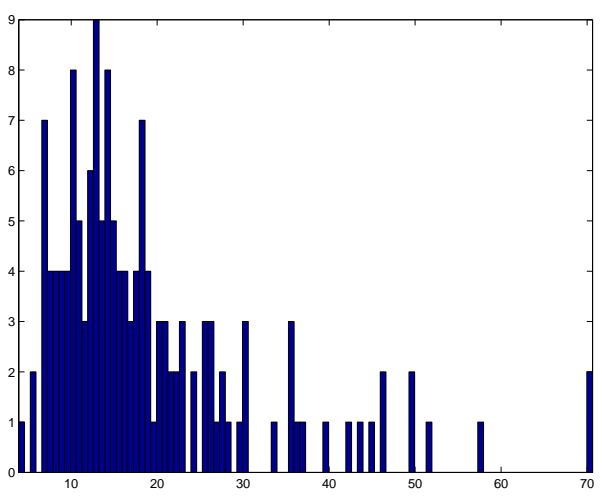

(d) volatility S\&P 500 - cojump days

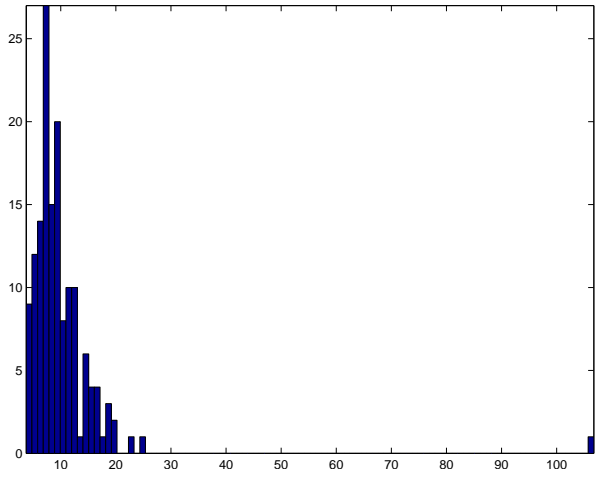

(f) volatility bonds- cojump days

Figure 15: S\&P 500 and Bonds daily volatility and correlation distributions: unconditional vs. cojump days 


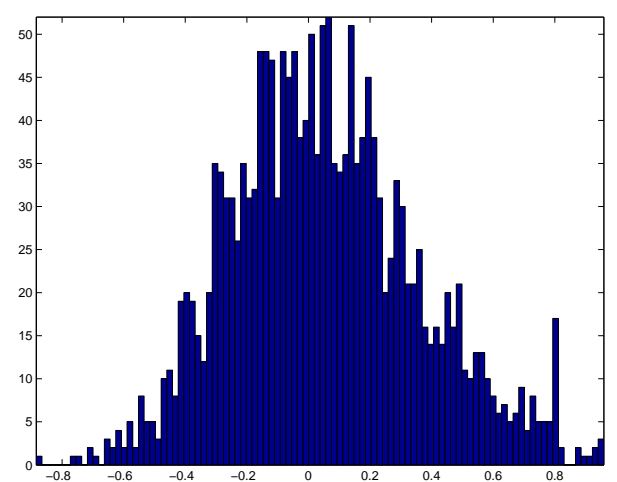

(a) correlation

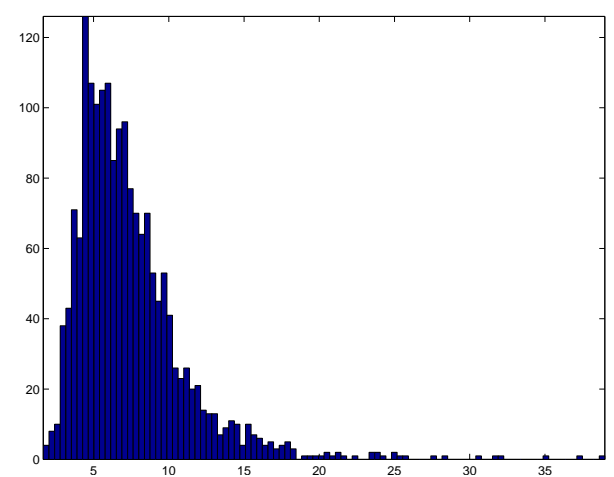

(c) volatility JPY/USD

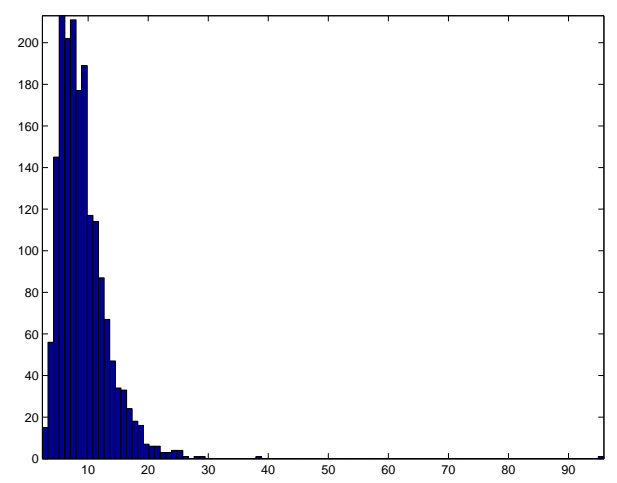

(e) volatility bonds

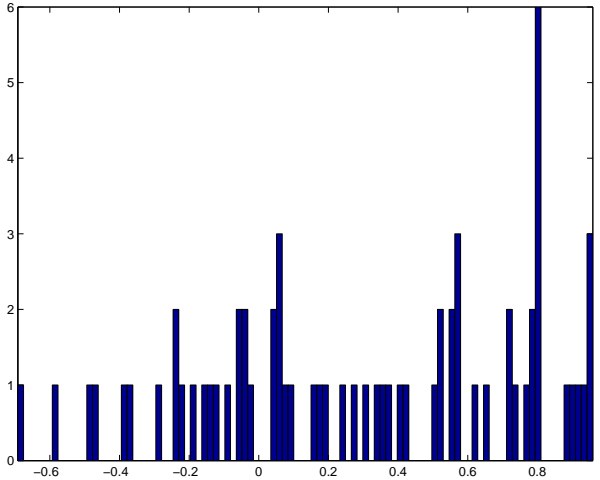

(b) correlation - cojump days

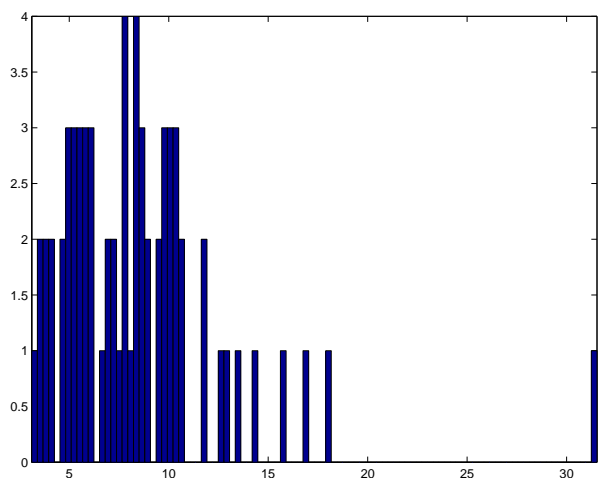

(d) volatility JPY/USD - cojump days

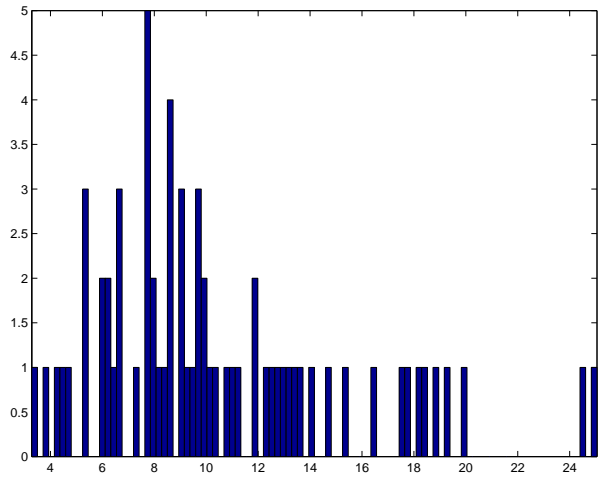

(f) volatility bonds- cojump days

Figure 16: JPY/USD and Bonds daily volatility and correlation distributions: unconditional vs. cojump days 